\title{
The cycle of interstellar dust in galaxies of different morphological types
}

\author{
F. Calura ${ }^{1}$, A. Pipino ${ }^{2,3}$, and F. Matteucci ${ }^{1,2}$ \\ 1 INAF - Osservatorio Astronomico di Trieste, via G. B. Tiepolo 11, 34131 Trieste, Italy \\ e-mail: fcalura@oats.inaf.it \\ 2 Dipartimento di Astronomia - Universitá di Trieste, via G. B. Tiepolo 11, 34131 Trieste, Italy \\ 3 Astrophysics, University of Oxford, Denys Wilkinson Building, Keble Road, Oxford OX1 3RH, UK
}

Received 14 June 2007 / Accepted 8 September 2007

\section{ABSTRACT}

\begin{abstract}
Aims. We used chemical evolution models for galaxies of different morphological type to perform a detailed study of the evolution of the cosmic dust properties in different environments: the solar neighbourhood, elliptical galaxies and dwarf irregular galaxies. Thanks to the uptodate observations available in the solar vicinity, we intend to study the effects of dust in the chemical evolution of different types of galaxies and, at the same time, to refine investigation of the parameter space to satisfactorily fine-tune the parameters in our study.

Methods. We have considered dust production from low and intermediate mass stars, supernovae Ia, supernovae II, and both dust destruction and dust accretion processes in a detailed model of chemical evolution for the solar vicinity. Then, by means of the same dust prescriptions, but adopting different galactic models (different star formation histories and the presence of galactic winds), we extended our study to ellipticals and dwarf irregular galaxies. In all these systems, dust evolution was calculated by means of chemical evolution models that relax the instantaneous recycling approximation and already reproduce the main features of the various galaxies. Results. We have investigated how the assumption of different star formation histories affects the dust production rates, dust depletion, the dust accretion, and destruction rates. We predict dust-to-gas and dust-to-metal ratios in very good agreement with those observed in the solar vicinity. We show how the inclusion of the dust treatment is helpful in solving the so-called Fe discrepancy, as observed in the hot gaseous halos of local ellipticals, and in reproducing the chemical abundances observed in the Lyman Break Galaxies. Finally, our new models can be very useful in future detailed spectro-photometric studies of galaxies.
\end{abstract}

Key words. ISM: dust, extinction - ISM: abundances - galaxies: abundances - galaxies: evolution

\section{Introduction}

The presence of dust in local and distant galaxies is indicated by various observational proofs. The light emitted by stars interacts with the dust grains in a wavelength-dependent manner, as the light emitted in the ultra-violet and optical bands is absorbed and scattered by the dust grains. This is the well-known phenomenon called dust extinction, which is taking place both inside and outside galaxies (Aguirre 1999). In the optical band, the larger the emission wavelength, the lower the extinction effect caused by dust.

In our Galaxy and in local starburst galaxies, dust grains are the main contributors to the emission in the mid and far infrared bands. In fact, the light absorbed by dust at UV and optical wavelengths is then thermally re-emitted at much longer wavelengths, in the range $10-1000 \mu \mathrm{m}$. Also the chemical composition of the galactic interstellar medium (ISM) is strongly influenced by the presence of dust. Some chemical elements, called refractory, in the gas phase are subject to dust depletion, and a fraction of their total abundance is incorporated into solid grains. Examples of refractory elements are $\mathrm{Fe}, \mathrm{Si}, \mathrm{Mg}, \mathrm{Ni}$. For these elements, the abundance measured in stellar surfaces is considerably higher than the gas phase abundances ( $\mathrm{Li} 2005$ ).

Dwek (1998, hereinafter D98) has developed a chemical evolution model to study the dust content of the Milky Way galaxy and its evolution. He focused on some refractory chemical species and for each one suggested a set of dust condensation efficiencies, calculating the dust production rates from low and intermediate mass stars, and from type Ia and type II supernovae (SNe). For these elements, he also investigated the dust destruction and the accretion rates, the dust-to-gas ratios, and the dust fractions at the present time.

Since then, the amount of observational data regarding the local content of dust has grown noticeably (Draine 2003), allowing a reliable fine-tuning of the parameters involved in theoretical dust-evolution studies. By means of a one-zone chemical evolution for the solar neighbourhood, Zhukovska et al. (2007) studied production by stars of various dust species. By considering the available observations of presolar dust grains in meteorites, their study allowed them to put some constraints on the dust condensation efficiencies from $\mathrm{SNe}$ and AGB stars. Moving to extragalactic objects, new observations call for a theoretical investigation of the dust content of galaxies of different morphological types. In particular, the SCUBA camera revealed a large number of dusty sources at high redshfit, and these might be young massive ellipticals undergoing a starburst (Lilly et al. 1999; Eales et al. 2000).

For what concerns, instead, local ellipticals, new measurements have rejuvenated the interest in assessing the issue of the so-called iron-discrepancy (Arimoto et al. 1997) by means of the dust. In this paper, we present a new set of chemical evolution models, with updated nucleosynthesis prescriptions. All of these models are successful in reproducing the abundances of various 
galaxies (spirals, ellipticals, irregulars). By means of our chemical evolution models, we studied the dust evolution in various environments. Starting from the same formalism developed by D98, we first focus on the solar neighbourhood (S.N.) and extend the analysis by D98, improving and deepening the investigation of the parameter space. We focus on the differential roles of type Ia and II SNe in dust destruction and production and on the evolution of the accretion and destruction rates. When possible, we compare these quantities with the data available in the literature computed by other authors. In this paper, we perform a chemical evolution study of various dust species in environments that are different from the Milky Way Galaxy. This is possible by taking the stellar lifetimes into account in detail, thereby allowing us to predict the properties of dust species, such as $\mathrm{C}$ and $\mathrm{Fe}$, produced by stars with lifetimes spanning from $\sim 0.03$ Gyr up to $\sim 10$ Gyr. We focus on the effects that different star formation histories have on the evolution of the dust content of galaxies with different morphological types.

This paper is organised as follows. In Sect. 2 we present the chemical evolution equations used to compute dust evolution and all the parameters involved. In Sect. 3 we discuss our results for the S.N., for elliptical galaxies, and for dwarf irregular galaxies. Finally, in Sect. 4 we draw some conclusions.

\section{The chemical evolution model}

\subsection{The equations}

By means of detailed chemical evolution models, it is possible to follow the evolution of the abundances of several chemical species and the dust content of galaxies of different morphological type. The instantaneous recycling approximation is relaxed in all models and the stellar lifetimes are taken into account. Detailed descriptions of the chemical evolution models can be found in Matteucci \& Tornambé (1987) and Matteucci (1994) for elliptical galaxies, in Chiappini et al. $(1997,2001)$ for the spirals, and in Bradamante et al. (1998) for irregular galaxies.

In our picture, elliptical galaxies form as a result of the rapid collapse of a homogeneous sphere of primordial gas where intense star formation (SF) is taking place at the same time as the collapse proceeds. The SF is assumed to halt as the energy of the ISM, heated by stellar winds and supernova (SN) explosions, exceeds the binding energy of the gas. At this time a galactic wind occurs, sweeping away almost all of the residual gas. After the $\mathrm{SF}$ has stopped, the galactic wind is maintained by type Ia $\mathrm{SNe}$, and its duration depends on the balance between this heating source and the gas cooling (we refer the reader to Pipino et al. 2002, 2005).

For spiral galaxies, the adopted model is calibrated in order to reproduce a large set of observational constraints for the Milky Way galaxy (Chiappini et al. 2001). The Galactic disc is approximated by several independent rings, $2 \mathrm{kpc}$ wide, without any exchange of matter between them. In our picture, spiral galaxies are assumed to form as a result of two main infall episodes. During the first episode, the halo and the thick disc are formed. During the second episode, a slower infall of external gas forms the thin disc with the gas accumulating faster in the inner than in the outer region ("inside-out" scenario, Matteucci \& François 1989). The process of disc formation is much longer than the halo and bulge formation, with time scales varying from $\sim 2 \mathrm{Gyr}$ in the inner disc to $\sim 7 \mathrm{Gyr}$ in the solar region and up to $20 \mathrm{Gyr}$ in the outer disc (see Table 1). In this paper, we are interested in the study of dust evolution in the S.N. For this purpose, we focus on a ring located at $8 \mathrm{kpc}$ from the Galactic centre, $2 \mathrm{kpc}$ wide.
Finally, irregular galaxies are assumed to assemble from infall of protogalactic small clouds of primordial chemical composition, until masses in the range $\sim 10^{9} M_{\odot}$ are accumulated, and to produce stars at a lower rate than spirals.

All the models used in this paper consider only one gas phase. For a single-phase gas, the chemical evolution equation for a given chemical element $i$ takes the following form:

$$
\begin{aligned}
\frac{\mathrm{d} G_{i}(t)}{\mathrm{d} t}= & -\psi(t) X_{i}(t) \\
& +\int_{M_{L}}^{M_{B_{m}}} \psi\left(t-\tau_{m}\right) Q_{\mathrm{mi}}\left(t-\tau_{m}\right) \phi(m) \mathrm{d} m \\
& +A \int_{M_{B_{m}}}^{M_{B_{M}}} \phi(m) \\
& \times\left[\int_{\mu_{\min }}^{0.5} f(\mu) Q_{\mathrm{mi}}\left(t-\tau_{m_{2}}\right) \psi\left(t-\tau_{m_{2}}\right) \mathrm{d} \mu\right] \mathrm{d} m \\
& +(1-A) \int_{M_{B_{m}}}^{M_{B_{M}}} \psi\left(t-\tau_{m}\right) Q_{\mathrm{mi}}\left(t-\tau_{m}\right) \phi(m) \mathrm{d} m \\
& +\int_{M_{B_{M}}}^{M_{U}} \psi\left(t-\tau_{m}\right) Q_{\mathrm{mi}}\left(t-\tau_{m}\right) \phi(m) \mathrm{d} m \\
& +\left(\frac{\mathrm{d} G_{i}(t)}{\mathrm{d} t}\right)_{\mathrm{inf}}-\left(\frac{\mathrm{d} G_{i}(t)}{\mathrm{d} t}\right)_{\mathrm{out}}
\end{aligned}
$$

where $G_{i}(t)=M_{\mathrm{g}}(t) X_{i}(t) / M_{\mathrm{tot}}$ is the gas mass in the form of an element $i$ normalized to a total fixed mass $M_{\text {tot }}$, and $G(t)=$ $M_{\mathrm{g}}(t) / M_{\text {tot }}$ is the total fractional mass of gas in the galaxy at the time $t$. The same quantities can be defined in terms of the surface gas $\left(\sigma_{\text {gas }}\right)$ and mass $(\sigma)$ densities, especially in spiral galaxies. Here, $X_{i}(t)$ is defined as the abundance by mass (or mass fraction) of the element $i$ (for a comprehensive discussion of this equation, see Matteucci \& Greggio 1986). The quantity $\psi(t)$ is the star formation rate (SFR). The term $-\psi(t) X_{i}(t)$ gives the rate at which the element $i$ is subtracted from the ISM by the SF process. The second term is the rate at which each element is restored into the ISM by single stars with masses in the range $M_{L}-M_{B_{m}}$, where $M_{L}$ is the minimum mass contributing, at a given time $t$, to chemical enrichment (the minimum is $0.8 M_{\odot}$ ) and $M_{B_{m}}$ is the minimum binary mass allowed for binary systems giving rise to type Ia SN (3 $M_{\odot}$, Matteucci \& Greggio 1986). The quantities $Q_{m i}\left(t-\tau_{m}\right)$ (where $\tau_{m}$ is the lifetime of a star of mass $m$ ) contain all the information about stellar nucleosynthesis for elements either produced or destroyed inside stars or both (Talbot \& Arnett 1971). The third term represents the enrichment due to binaries that become type Ia SN, i.e. all the binary systems with total mass between $M_{B_{m}}$ and $M_{B_{M}}=16 M_{\odot}$. For the type Ia SN progenitor model, the single degenerate (SD) scenario is assumed, where a C-O white dwarf explodes by $\mathrm{C}$-deflagration mechanism after having reached the Chandrasekhar mass $\left(1.44 M_{\odot}\right)$, owing to progressive mass accretion from a non-degenerate companion (Whelan $\&$ Iben 1973). The parameter $A$ represents the unknown fraction of binary stars giving rise to type Ia SN and is fixed by reproducing the observed present time SN Ia rate. In this third term, both quantities $\psi$ and $Q_{\mathrm{mi}}$ refer to the time $t-t_{m_{2}}$, where $t_{m_{2}}$ indicates the lifetime of the secondary star of the binary system, which regulates the explosion timescale. The quantity $\mu=M_{2} / M_{B}$ is the ratio between the mass of the secondary component $M_{2}$ and the total mass of the binary system $M_{B}$, whereas $f(\mu)$ is the 
distribution function of this ratio. Statistical studies indicate that mass ratios close to 0.5 are preferred, so the formula

$$
f(\mu)=2^{1+\gamma}(1+\gamma) \mu^{\gamma}
$$

is commonly adopted, with $\gamma=2$ (Greggio \& Renzini 1983). Here, $\mu_{\min }$ is the minimum mass fraction contributing to the SNIa rate at the time $t$ and is given by

$\mu_{\min }=\max \left\{\frac{M_{2}(t)}{M_{B}}, \frac{M_{2}-0.5 M_{B}}{M_{B}}\right\}$.

The fourth term represents the enrichment due to stars in the mass range $M_{B_{m}}-M_{B_{M}}$, which are either single or, if in binaries, do not produce a SN Ia event. In this mass range, all the stars with masses $m>8 M_{\odot}$ will explode as type II SNe, which in our picture are assumed to stem from the core collapse of single massive stars. The fifth term represents the enrichment of stars more massive than $M_{B_{M}}$, all of which explode as corecollapse $\mathrm{SNe}$. As the upper mass limits contributing to the chemical enrichment, we assume $M_{U}=100 M_{\odot}$. Finally, the last two terms account for infall of external gas and for galactic winds, respectively. For the infall term, an exponential law with different timescales is adopted for spirals and irregulars (see Calura \& Matteucci 2006b). Concerning ellipticals, Pipino \& Matteucci (2004) have shown that, in order to satisfy the largest number of photo-chemical properties, a quick infall is needed with a shorter timescale for the more massive objects.

A non-zero outflow term is present in the equations describing ellipticals and irregular galaxies. In both of these galaxies, a galactic wind develops as the thermal energy of the gas heated by SN explosions exceeds the binding energy of the gas (see Bradamante et al. 1998; Pipino \& Matteucci 2004). The binding energy of the gas is strongly influenced by assumptions concerning the presence and distribution of dark matter (Matteucci 1992). For the model adopted here, a diffuse $\left(R_{\mathrm{e}} / R_{\mathrm{d}}=0.1\right.$, where $R_{\mathrm{e}}$ is the effective radius of the galaxy and $R_{\mathrm{d}}$ the radius of the dark matter core $)$, massive $\left(M_{\text {dark }} / M_{\text {lum }}=10\right)$ dark halo has been assumed (see Bertin et al. 1992). In the case of spiral galaxies, the outflow term is set to zero.

\subsubsection{The initial mass function}

The initial mass function (IMF) $\phi(m)$ is assumed to be constant in space and time and normalised to unity in the mass interval 0.1-100 $M_{\odot}$. For the spiral galaxies, we adopt a simplified twoslope approximation to the actual Scalo (1986) IMF, similar to what was done in Matteucci \& François (1989), which is expressed by the formula

$\phi_{\text {Scalo }}(m)= \begin{cases}0.19 \cdot m^{-1.35} & \text { if } m<2 M_{\odot} \\ 0.24 \cdot m^{-1.70} & \text { if } m>2 M_{\odot},\end{cases}$

whereas for ellipticals and irregulars we adopt a Salpeter (1995) IMF with the form

$\phi_{\text {Salp }}(m)=0.17 \cdot m^{-1.35}$.

The reason for such a choice relies mainly on the chemical abundances and on the metal content observed in each morphological type (see Calura \& Matteucci 2006b). In fact, it is well known that a Salpeter (1955) IMF can account for the abundances observed in local ellipticals and dwarf galaxies (Pipino \& Matteucci 2004; Recchi et al. 2002), whereas it leads to an overestimation of the metal abundances in spiral discs (see Romano et al. 2005), which, on the other hand, are well accounted for by means of the Scalo (1986) IMF.

\subsubsection{The nucleosynthesis prescriptions}

The yields used in the present work are separated into three groups: those of low and intermediate mass stars, of type Ia SNe, and yields of massive stars. Low and intermediate mass stars (i.e. with masses $0.8 M_{\odot} \leq m \leq 8 M_{\odot}$ ) contribute through quiescent mass loss and planetary nebula phase to the ISM metal enrichment. Type Ia SNe are assumed to originate from exploding white dwarfs in binary systems, characterised by total masses $3 M_{\odot} \leq M_{\mathrm{B}} \leq 16 M_{\odot}$, according to the Matteucci \& Recchi (2001) best model. Finally, we assume that single massive stars with initial masses in the range $>8-100 M_{\odot}$ explode as core collapse $\mathrm{SNe}$. The nucleosynthesis prescriptions are common to all models. For massive stars and type Ia SNe, we adopt the empirical yields suggested by François et al. (2004), which are substantially based on the Woosley \& Weaver (1995) and Iwamoto et al. (1999) yields, respectively, and are tuned to best reproduce the abundances in the S.N. For low and intermediate mass stars, we adopt the prescriptions by van den Hoek \& Groenewegen (1997).

\subsubsection{The star formation rate}

For ellipticals and irregulars galaxies, the SFR $\psi(t)$ in our models is a Schmidt (1959) law expressed as

$\psi(t)=v G^{k}(t)$

The quantity $v$ is the efficiency of SF, namely the inverse of the typical time scale for SF and is expressed in $\mathrm{Gyr}^{-1}$.

In the case of spiral galaxies, the SFR expression (Chiappini et al. 1997) is

$\psi(r, t)=v\left[\frac{\sigma(r, t)}{\sigma\left(r_{\odot}, t\right)}\right]^{2(k-1)}\left[\frac{\sigma\left(r, t_{\mathrm{Gal}}\right)}{\sigma(r, t)}\right]^{k-1} G^{k}(r, t)$

where $v$ is the SF efficiency, $\sigma(r, t)$ the total mass (gas + stars) surface density at a radius $r$ and time $t, \sigma\left(r_{\odot}, t\right)$ is the total mass surface density in the solar region. For the gas density exponent $k$ a value of 1.5 has been assumed by Chiappini et al. (1997) in order to ensure a good fit to the observational constraints for a large set of local spirals (Kennicutt 1998). The efficiency of SF is set to $v=1 \mathrm{Gyr}^{-1}$, and becomes zero when the gas surface density drops below a certain critical threshold. For the SF, we adopt a threshold gas density $\sigma_{\text {th }} \sim 7 M_{\odot} \mathrm{pc}^{-2}$ in the disc as suggested by Kennicutt (1989). The difference between Eqs. (4) and (5) is due to the different normalisations of the quantities involved in the chemical evolution equations (see Sect. 2.1). Furthermore, the model used for spirals is a multi-zone one, where the SFR expression is a function of the galactocentric radius. On the other hand, the models of ellipticals and irregulars are one-zone and the SFR has a simpler expression. It is important to note that the parameterization of the SFR in both Eqs. (4) and (5) involves two different quantities, i.e. $v$ and $k$, which act in the same way.

In Table 1, we show the adopted parameters for all the chemical evolution models described in this section. The various models are listed in Col. 1. In Cols. 2-6, we present the adopted parameters, i.e. the SF efficiency $v$, the infall timescale $\tau_{\text {inf }}$, the total surface mass density $\sigma_{\text {tot }}$ (in the case of the Milky Way), or the baryonic mass $M_{\text {lum }}$ (in the case of the elliptical/irregular model), the Schmidt law exponent $k$ and the IMF, respectively.

In Fig. 1, we show different SFRs for various models used in this work. In panel (a), we show the SFRs for the two models considered here for spiral galaxies: the S.N. model and a model for the disc at a distance of $16 \mathrm{kpc}$ from the Galactic centre. 
The most striking features of the S.N. model are the SF hiatus at 1 Gyr (see Chiappini et al. 1997, 2001) and the thresholddominated SF after 10 Gyr. Both features have big consequences on the behaviour of several quantities studied in this paper. The model used for ellipticals is the one by Pipino et al. (2005). This is a first step in the self-consistent study of both optical and X-ray properties of elliptical galaxies by means of a chemical evolution code. Detailed cooling and heating processes in the ISM are taken into account using a mono-phase, one-zone treatment, giving us a reliable modelling of the galactic wind regime. For reproducing a giant elliptical, we make use of the Pipino et al. (2005) case Ha1, which is characterised by a luminous mass $M_{\text {lum }}=10^{12} M_{\odot}$, with as SF efficiency $v=25 \mathrm{Gyr}^{-1}$ and an infall timescale $\tau=0.2 \mathrm{Gyr}$, in which a mild secular gas accretion from the surrounding IGM is allowed. For a more typical spheroid $\left(L \sim L_{*}\right)$, we refer to their model La1, which has a luminous mass $M_{\text {lum }}=10^{11} M_{\odot}$, as SF efficiency $v=15 \mathrm{Gyr}^{-1}$ and an infall timescale $\tau=0.3 \mathrm{Gyr}$. We stress that, due to the uptodate nucleosynthesis prescriptions and to the inclusion of dust, the models presented here are intended as an improved version of the original Pipino et al. (2005) models Ha1 and La1. To study the properties of a Lyman Break Galaxy, we use a model characterised by a luminous mass $M_{\text {lum }}=10^{10} M_{\odot}$, an SF efficiency $v=5 \mathrm{Gyr}^{-1}$, and an infall timescale $\tau=0.5 \mathrm{Gyr}$, referred to as the LBGa1 model. In our scheme, large galaxies form the bulk of their stars in a shorter timescale than small galaxies, according to the "inverse wind" scenario. As shown by Matteucci (1994), this scenario allows us to reproduce the correlation between the $[\mathrm{Mg} / \mathrm{Fe}]$ ratio and the galactic mass observed in local ellipticals.

In panel (b), we show the predicted SF history for two models of elliptical galaxies (La1 and Ha1), characterised by very high SFR values (from $\sim 200$ to $\sim 3000 M_{\odot} / \mathrm{yr}$ ) and by a starburst lasting 0.4-0.9 Gyr. After this strong event, the SF stops and a galactic wind sets in for several Gyrs, until a diffuse and hot gaseous halo, which surrounds the galaxy, is formed (for a more detailed description of this model, see Pipino et al. 2005).

Finally, in panel (c) of Fig. 1, we show two different models tested for an irregular galaxy: an irregular dwarf with continuous SF (IC) and a starburst irregular. For the IC model, we assume continuous SFR with SF efficiency of $v_{\mathrm{IC}}=0.05 \mathrm{Gyr}^{-1}$. On the other hand, the starburst model is similar to model B used by Lanfranchi \& Matteucci (2003). The SFR of this model consists of 3 bursts with an efficiency of $v_{\text {burst }}=0.5 \mathrm{Gyr}^{-1}$. The occurrence times of the bursts are $1 \mathrm{Gyr}, 10 \mathrm{Gyr}$, and 13 Gyr. The durations for each burst are $0.02 \mathrm{Gyr}, 0.02 \mathrm{Gyr}$, and $0.2 \mathrm{Gyr}$, respectively (for more details, see Lanfranchi \& Matteucci 2003). The IC and burst models have been used to reproduce the abundance patterns observed in damped Lyman alpha (DLA) systems (Calura et al. 2003; Dessauges-Zavadsky et al. 2004) and allow us to reproduce the present-day features of local dwarf irregulars and blue compact galaxies (Recchi et al. 2002; Lanfranchi \& Matteucci 2003), respectively. This latter model should be regarded as representative of a blue compact dwarf galaxy. Our assumption is based on the results by Lanfranchi \& Matteucci (2003), who have shown that the N/O, C/O, Si/O, and O/Fe ratios observed in BCGs can be explained by a model with two to seven short bursts of SF with efficiencies in the range $v=0.1-0.9 \mathrm{Gyr}^{-1}$

\subsection{Chemical evolution of the dust}

The chemical evolution of an element $i$ in the dust is computed by using the formalism developed by D98. Let $X_{\text {dust, } i}(t)$ be the
Table 1. Adopted parameters for the galactic models used in this work.

\begin{tabular}{lccccc}
\hline \hline \multicolumn{1}{c}{ Model } & \multicolumn{5}{c}{ Parameters } \\
& $\begin{array}{c}v \\
\left(\mathrm{Gyr}^{-1}\right)\end{array}$ & $\begin{array}{c}\tau_{\text {inf }} \\
(\mathrm{Gyr})\end{array}$ & $\begin{array}{c}\sigma_{\text {tot }} \\
\left(M_{\odot} / \mathrm{pc}^{2}\right)\end{array}$ & $k$ & IMF \\
\hline Milky Way, S.N. & 1 & 7 & 54 & 1.5 & Scalo \\
Milky Way, 16 kpc & 1 & 20 & 5 & 1.5 & Scalo \\
\hline \multicolumn{1}{c}{ Model } & $v$ & $\tau_{\text {inf }}$ & $M_{\text {lum }}$ & $k$ & IMF \\
& $\left(\mathrm{Gyr}^{-1}\right)$ & $(\mathrm{Gyr})$ & $\left(M_{\odot}\right)$ & & \\
\hline Elliptical, La1 & 15 & 0.3 & $10^{11}$ & 1 & Salpeter \\
Elliptical, Ha1 & 25 & 0.2 & $10^{12}$ & 1 & Salpeter \\
Irregular, IC & 0.05 & 10 & $10^{9}$ & 1 & Salpeter \\
Irregular, burst & 0.5 & 10 & $10^{9}$ & 1 & Salpeter \\
\hline
\end{tabular}

abundance by mass of element $i$ in the dust, and $G(t)$ the ISM fraction at the time $t$. The quantity

$G_{\text {dust }, i}(t)=X_{\text {dust }, i} \cdot G(t)$

represents the normalised mass density of element $i$ at time $t$ in the dust. The time evolution of $G_{\text {dust }, i}(t)$ is calculated by means of the equation

$$
\begin{aligned}
& \frac{\mathrm{d} G_{\mathrm{dust}, i}(t)}{\mathrm{d} t}=-\psi(t) X_{\mathrm{dust}, i}(t) \\
& +\int_{M_{L}}^{M_{B_{m}}} \psi\left(t-\tau_{m}\right) \delta_{i}^{\mathrm{SW}} Q_{\mathrm{mi}}\left(t-\tau_{m}\right) \phi(m) \mathrm{d} m \\
& +A \int_{M_{B_{m}}}^{M_{B_{M}}} \phi(m) \\
& \times\left[\int_{\mu_{\mathrm{min}}}^{.5} f(\mu) \psi\left(t-\tau_{m 2}\right) \delta_{i}^{\mathrm{Ia}} Q_{\mathrm{mi}}\left(t-\tau_{m 2}\right) \mathrm{d} \mu\right] \mathrm{d} m \\
& +(1-A) \int_{M_{B_{m}}}^{8 M_{\odot}} \psi\left(t-\tau_{m}\right) \delta_{i}^{\mathrm{SW}} Q_{\mathrm{mi}}\left(t-\tau_{m}\right) \phi(m) \mathrm{d} m \\
& +(1-A) \int_{8 M_{\odot}}^{M_{B_{M}}} \psi\left(t-\tau_{m}\right) \delta_{i}^{I I} Q_{\mathrm{mi}}\left(t-\tau_{m}\right) \phi(m) \mathrm{d} m \\
& +\int_{M_{B_{M}}}^{M_{U}} \psi\left(t-\tau_{m}\right) \delta_{i}^{I I} Q_{\mathrm{mi}}\left(t-\tau_{m}\right) \phi(m) \mathrm{d} m \\
& -\frac{G_{\mathrm{dust}, i}}{\tau_{\mathrm{destr}}}+\frac{G_{\mathrm{dust}, i}}{\tau_{\mathrm{accr}}}-\left(\frac{\mathrm{d} G_{\mathrm{dust}, i}(t)}{\mathrm{d} t}\right)_{\mathrm{out}}
\end{aligned}
$$

The main differences between Eqs. (1) and (7) concern all the integrals on the right hand sides of both equations. These integrals, in the case of Eq. (7) contain the quantities $\delta_{i}^{\mathrm{SW}}, \delta_{i}^{\mathrm{Ia}}$, and $\delta_{i}^{I I}$, i.e. the condensation efficiencies of the element $i$ in stellar winds, type Ia SNe, and type II SNe (see Sect. 2.2.1). These quantities represent the fractions of the element $i$ which is condensed into dust and restored into the ISM by low and intermediate mass stars, type Ia SNe, and type II SNe, respectively. The seventh and eighth terms of Eq. (7) represent the dust destruction and accretion rates, respectively. These terms depend on the quantities $\tau_{\text {destr }}$ and $\tau_{\text {accr }}$, which represent the typical timescales for destruction and accretion, respectively. These two quantities are discussed in detail in Sects. 2.2.2 and 2.2.3. Finally, the last term of Eq. (7) accounts for possible ejection of dust into the inter galactic medium (IGM) by means of galactic winds. This term is absent in the equation for the S.N. model, but is taken into account in the elliptical and irregular models. 

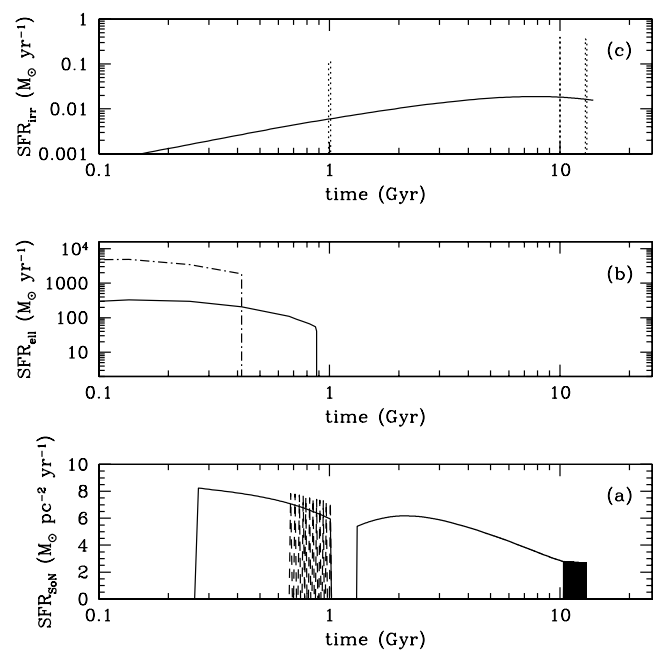

Fig. 1. Predicted SFRs as a function of time for different chemical evolution models. Panel a): S.N. (solid line) and a model for the outer regions of the Milky Way disc (dashed line); panel b): two different elliptical galaxy models, i.e. model La1 (solid line) and Ha1 (dash-dotted line) of Pipino et al. (2005); panel c): two different irregulars, one with continuous SF (solid line) and the other with 3 starbursts (dotted line).

\subsubsection{Dust condensation efficiencies}

We assume the dust condensation efficiencies suggested by D98. The choice of the following dust condensation efficiencies is motivated by various lines of evidence, as discussed in detail in D98. In this paper, we focus on the main refractory elements that are depleted into dust in the cold phase. These elements are $\mathrm{C}, \mathrm{O}, \mathrm{Mg}, \mathrm{Si}, \mathrm{S}, \mathrm{Ca}, \mathrm{Fe}$. As an assumption, we consider that only these elements can be incorporated into dust grains. We assume that dust grains can be of two types: carbon (C) dust and silicate ( $\mathrm{Si}$ ) dust. We are aware that dust composition can be more complicated. For instance, Mathis (1996) presented a model that also includes the possibility of composite grains containing carbon, silicates, and oxides. Li \& Greenberg (1997) present a trimodal dust model with large silicate core-organic refractory mantle dust particles, small carbonaceous particles, and Polycyclic aromatic hydrocarbon particles able to reproduce the interstellar extinction and polarisation observational constraints. Furthermore, different dust structures would also have an impact on dust destruction and accretion (see Greenberg \& Li 1999; Jones et al. 1996). However, the inclusion of more complicated dust types does not fit the aims of this paper, since it would increase the number of free parameters involved in our study. According to the formalism developed in D98, the contributors to the dust production are (a) low and intermediate mass stars, (b) type Ia SNe, and (c) type II SNe. We neglect the contributions from Wolf-Rayet stars and novae (D98), both of which are believed to represent unimportant sources of dust production.

a. Low and intermediate mass stars - In these stars, dust is produced during the asymptotic giant branch (AGB) phase (Ferrarotti \& Gail 2006, and references therein). We assume that dust formation depends mainly on the composition of the stellar envelopes. If $X_{\mathrm{O}}$ and $X_{\mathrm{C}}$ represent the $\mathrm{O}$ and $\mathrm{C}$ mass fractions in the stellar envelopes, respectively, we assume that stars with $X_{\mathrm{O}} / X_{\mathrm{C}}>1$ are producers of silicate dust, i.e. dust particles composed by $\mathrm{O}, \mathrm{Mg}, \mathrm{Si}, \mathrm{S}, \mathrm{Ca}$, and $\mathrm{Fe}$. On the other hand, C-rich stars, characterised by $X_{\mathrm{O}} / X_{\mathrm{C}}<1$, are producers of carbonaceous solids, i.e. carbon dust (Draine 1990). Since $M_{i, \mathrm{ej}}(m)$ and $M_{i, \mathrm{dust}}(m)$ are the total ejected mass and the dust mass formed by the stars as functions of the initial mass $m$ for the element $i$, respectively, we assume that for stars with $X_{\mathrm{O}} / X_{\mathrm{C}}<1$

$$
\begin{aligned}
& M_{\text {dust }, \mathrm{C}}(m)=\delta_{\mathrm{C}}^{\mathrm{SW}} \cdot\left[M_{C, \mathrm{ej}}(m)-0.75 M_{O, \mathrm{ej}}(m)\right] \\
& \text { with } \delta_{\mathrm{C}}^{\mathrm{SW}}=1 \text { and } \\
& M_{\text {dust }, i}(m)=0
\end{aligned}
$$

for all the other elements. For stars with $X_{\mathrm{O}} / X_{\mathrm{C}}>1$ in the envelope, we assume

$$
\begin{aligned}
& M_{\text {dust }, \mathrm{C}}(m)=0 \\
& M_{\text {dust }, i}(m)=\delta_{i}^{\mathrm{SW}} M_{i, \mathrm{ej}}(m) \\
& \text { with } \delta_{i}^{\mathrm{SW}}=1 \text { for } \mathrm{Mg}, \mathrm{Si}, \mathrm{S}, \mathrm{Ca}, \mathrm{Fe} \text { and } \\
& M_{\text {dust }, \mathrm{O}}(m)=16 \sum_{i} \delta_{i}^{\mathrm{SW}} M_{\mathrm{ej}, i}(m) / \mu_{i}
\end{aligned}
$$

with $\mu_{i}$ the mass of the $i$ element in atomic mass units.

b. Type Ia Supernovae - for these systems, we assume

$$
\begin{aligned}
& M_{\text {dust, } \mathrm{C}}(m)=\delta_{\mathrm{C}}^{\mathrm{Ia}}\left[M_{\mathrm{ej}, \mathrm{C}}(m)\right] \\
& \text { with } \delta_{\mathrm{C}}^{\mathrm{Ia}}=0.5
\end{aligned}
$$

$M_{\mathrm{dust}, i}(m)=\delta_{i}^{\mathrm{Ia}} M_{\mathrm{ej}, i}(m)$

with $\delta_{i}^{\mathrm{Ia}}=0.8$ for $\mathrm{Mg}, \mathrm{Si}, \mathrm{S}, \mathrm{Ca}, \mathrm{Fe}$;

$M_{\mathrm{dust}, \mathrm{O}}(m)=16 \sum_{i} \delta_{i}^{\mathrm{Ia}} M_{\mathrm{ej}, i}(m) / \mu_{i}$

b. Type II Supernovae - For type II SNe, we adopt the same prescriptions as for type Ia SNe. In a recent paper, Zhukovska et al. (2007) use a chemical evolution model for the solar neighbourhood to study the available observations of presolar dust grains in meteorites. Their analysis points towards lower condensation efficiencies than the ones suggested by D98, in particular for the silicates. In this paper, we test various assumptions concerning these parameters and study the effects on systems other than the solar neighbourhood.

\subsubsection{Dust destruction}

Dust destruction is primarily due to the propagation of SN shock waves in the warm/ionised interstellar medium (McKee 1989; Jones et al. 1994). Evidence of dust destruction comes from the observations of high-velocity clouds, where an anti-correlation between the depletion levels and the cloud velocities has been found (Shull 1978; McKee 1989). This was interpreted as evidence for grain destruction in SN shocks (D98). Following the suggestions by McKee (1989) and D98, for a given element $i$ the destruction timescale $\tau_{\text {destr }}$ can be expressed as

$\tau_{\mathrm{destr}, i}=\left(\epsilon M_{\mathrm{SNR}}\right)^{-1} \cdot \frac{\sigma_{\mathrm{gas}}}{R_{\mathrm{SN}}}$.

the destruction timescale is independent of the dust mass. As a result, $M_{\mathrm{SNR}}$ is the mass of the interstellar gas swept up by the SN remnant. For this quantity, McKee (1989) suggests a typical value of $M_{\mathrm{SNR}} \sim 6800 M_{\odot}$, which agrees with the results from numerical studies of SN evolution (Thornton et al. 1998). As suggested by McKee (1989), typical values for the destruction 
efficiency $\epsilon$ in a three-phase medium as the current local ISM are around 0.2 , hence we assume:

$\epsilon M_{\mathrm{SNR}}=0.2 \times 6800 M_{\odot}=1360 M_{\odot}$.

Here, $R_{\mathrm{SN}}$ is the total $\mathrm{SNe}$ rate, including the contributions by both type Ia and type II SNe. No significant modifications are required in a single-phase gas modelling (as in this paper, McKee 1989). We note, however, that McKee (1989) estimates $\epsilon M_{\mathrm{SNR}} \sim 70 M_{\odot}$ for a hot and rarefied medium as the gaseous halos surrounding ellipticals, therefore we run two models in which $\epsilon M_{\mathrm{SNR}}=1360 M_{\odot}$ until the galactic wind, and then we have an instantaneous transition to $\epsilon M_{\mathrm{SNR}} \sim 70 M_{\odot}$. The pre-wind dust evolution is obviously unaffected, whereas it leads to substantial changes in the late stage of the galactic evolution. According to the galaxy mass, we call them $L a l+M K$ and $H a l+M K$, respectively, and we show that they might produce interesting results, even though they use an over-simplified treatment of the dust destruction in the hot gas. Finally, to render the cases $\mathrm{Lal}$ and $\mathrm{Hal}$ more realistic, we further modified the destruction treatment by implementing a thermal sputtering term, which is thought to be the dominant source of dust destruction in hot plasmas. In particular, following Itoh (1989), we assume that nearly $90 \%$ of the dust grains will evaporate in a $\sim 1 \mathrm{keV}$ plasma by thermal sputtering in $\tau_{\text {dest }_{\mathrm{sp}}, i} \sim 10^{5} / n_{\mathrm{e}}\left(\mathrm{yr} \mathrm{cm}^{-3}\right)$, where the electron density $n_{\mathrm{e}}$ has been self-consistently evaluated at each timestep. This translates into a new destruction term, namely:

$\frac{G_{\mathrm{dust}, i}}{\tau_{\mathrm{destr}}}=G_{\mathrm{dust}, i}\left(70 M_{\odot}\right) \frac{R_{\mathrm{SN}}}{\sigma_{\mathrm{gas}}}+\frac{G_{\mathrm{dust}, i}}{\tau_{\mathrm{destr} \mathrm{sp}, i}}$.

According to the galaxy mass, we call the models featuring this particular term as Lal+Itoh and Hal+Itoh, respectively. For elliptical galaxies, these two models will be regarded as the fiducial ones. According to Itoh (1989), this term dominates the Fe grain evolution at late times.

In Sect. 3.1.2, we test the parameter $\epsilon$ and tune it in order to reproduce the depletion pattern observed in the S.N. Further refinements are presented in Sect. 3.2.

\subsubsection{Dust accretion}

Dust accretion occurs in dense molecular clouds, where volatile elements can condensate onto pre-existing grain cores, originating a volatile part called mantle (D98, Inoue 2003). Direct evidence for dust accretion comes from the observed large variations of the depletion levels as a function of the density (Savage $\&$ Sembach 1996) and from the observed infrared emission of cold molecular clouds (Flagey et al. 2006), which is charactesized by the absence of small-grain emission. These features can be accounted for by the coagulation of small grains on and into larger particles. Indirect evidence of dust accretion comes from the estimation of the grain lifetimes, which would be very small if no process could allow the grains to recondense and grow (McKee 1989; Draine \& Salpeter 1979). For a given element $i$, the accretion timescale $\tau_{\text {accr }}$ can be expressed as

$\tau_{\text {accr }}=\tau_{0, i} /\left(1-f_{i}\right)$,

where

$f_{i}=\frac{G_{\mathrm{dust}, i}}{G_{i}}$.

According to Eq. (11), the accretion timescale is an increasing function of the dust mass. For timescale $\tau_{0, i}$, typical values span



Fig. 2. Predicted dust production rates from various sources for a chemical evolution model of the S.N. In the lower (upper) panel, we show the results for the carbon (silicate) dust. Solid lines: contribution by low and intermediate mass stars (LIMS). Dotted lines: contribution by type II SNe. Dashed lines: contribution by type Ia SNe.

from $\sim 5 \times 10^{7} \mathrm{yr}$, of the order of the lifetime of a typical molecular cloud (D98), up to $\sim 2 \times 10^{8}$ yr (D98). In this paper, we assume that the timescale $\tau_{0, i}$ is constant for all elements, with a value of $5 \times 10^{7} \mathrm{yr}$.

In elliptical galaxies, we assume that dust accretion occurs only during the starburst epoch, when large amounts of cold gas and molecular $\mathrm{H}$ are available. After the onset of the wind and the end of the starburst, we assume that no molecular gas is present, hence no more accretion can occur. The observed molecular $\mathrm{H}$ content in dwarf irregular galaxies is very small, with molecular-to-atomic gas fractions of $\sim 10 \%$ or lower (Lisenfeld \& Ferrara 1998; Clayton et al. 1996). Motivated by these observational results, we assume that no accretion can occur in irregular galaxies.

\section{Results}

\subsection{Dust evolution in the Solar Neighbourhood}

\subsubsection{Dust production rates}

In Fig. 2, we show the predicted evolution of the carbon (lower panel) and silicate (upper panel) dust production rates calculated by means of the chemical evolution model for the S.N. In particular, we show the contributions to the rates by various sources: the low and intermediate mass stars (LIMS), the type II SNe, and the type Ia $\mathrm{SNe}$ (dashed lines). The production of the $\mathrm{C}$ dust is dominated by LIMS throughout most of the cosmic time. At present ( $\left.T_{0} \sim 13 \mathrm{Gyr}\right)$, a significant contribution is also coming from type II SNe, with type Ia $\mathrm{SNe}$ playing a negligible role. The frequent discontinuities in the lines are due to the effect of the star-formation threshold.

The production of silicate dust is dominated by type II SNe. At late times, the contributions by type Ia $\mathrm{SNe}$ and type II SNe are comparable. On the other hand, LIMS are negligible contributors to the Si dust production rate.

\subsubsection{The dust fractions}

An important test for the robustness of our formalism is represented by the study of the metal fractions in dust. These 


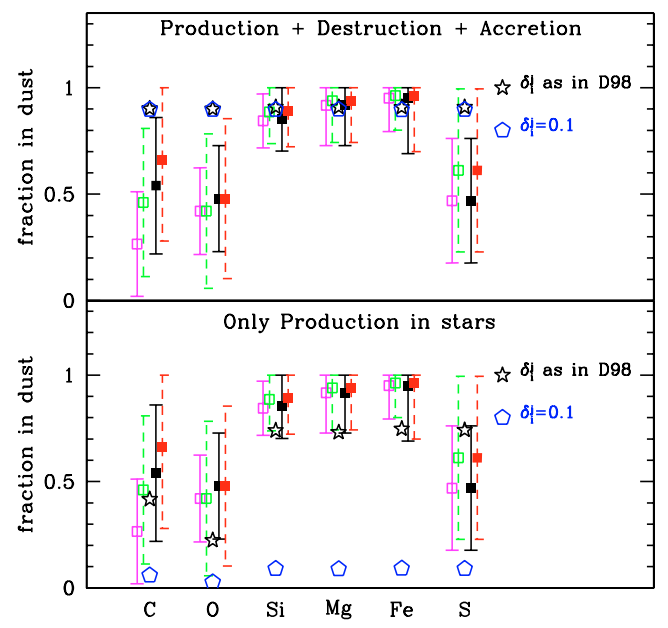

Fig. 3. Fractions in dust for various elements. Open stars: predicted present-day fractions calculated by adopting the prescriptions suggested by D98 for the dust condensation efficiencies. Open pentagons: predicted present-day fractions calculated by assuming a constant value of 0.1 for the dust condensation efficiencies. The solid and open squares are the fractions observed by Kimura et al. (2003) in the Local Interstellar Cloud using the set of cosmic abundances specified in their Tables 2 and 3, respectively. The squares with solid and dashed error bars have been calculated assuming a $\mathrm{H}$ ionization fraction of $\chi_{\mathrm{H}}=0.25$ and $\chi_{\mathrm{H}}=0.45$, respectively. In the lower panel, the predicted values are calculated considering only dust production in stars. In the upper panel, the predicted values are calculated considering dust production in stars, dust destruction by $\mathrm{SNe}$, and dust accretion in the ISM.

quantities are the ratios between the amount of a given element locked into dust and its total abundance and can be expressed as

$f_{i}=\frac{X_{\mathrm{dust}, i}}{X_{i}}$

Of particular interest is the comparison between the predicted dust fractions for various elements and the observed ones. This analysis is very useful to testing the various parameters involved in our study, in particular the dust condensation efficiencies $\delta_{i}^{\mathrm{SW}}, \delta_{i}^{\mathrm{Ia}}$, and $\delta_{i}^{\mathrm{II}}$, the dust destruction efficiency $\epsilon$, and the dust accretion timescale $\tau_{0, i}$, as well as the sensitivity of the results on the assumptions about these parameters. In Fig. 3 we show the predicted present-day fractions in dust for the elements studied in this work, compared to the values observed by Kimura et al. (2003) in the Local Interstellar Cloud. It is worth noting that the Local Interstellar Cloud consists of a warm medium, characterised by a temperature of $\sim 6000 \mathrm{~K}$, whereas our models provide the depletion of the cold gas, with temperatures lower than $\sim 100 \mathrm{~K}$. In this work, the dust fractions observed by Kimura et al. (2003) are taken as the reference. From the logarithmic depletions $\delta$ plotted in Fig. 2 of Kimura et al. (2003), it is possible to derive the dust fractions of the cold gas through the formula

$f=1-10^{\delta}$.

For $\mathrm{C}, \mathrm{O}$, and $\mathrm{S}$, the logarithmic depletions of the cold medium are very similar to the ones of the Local Intersetllar Cloud. For $\mathrm{Si}, \mathrm{Mg}$, and $\mathrm{Fe}$, the dust fractions of the cold medium are higher than the ones of the warm medium and are within the error bars plotted in Fig. 3. In the two panels of Fig. 3, the predictions are obtained by testing two different assumptions concerning the dust condensation efficiencies. In the lower panel of Fig. 3, we present the predicted values calculated considering only dust production in stars. The fractions are calculated by adopting for the dust condensation efficiencies both the prescriptions suggested by D98 and a constant value of 0.1 , regardless of the element and of the production source. Following the prescriptions suggested by D98, a higher amount of metals is locked into dust than with the other choice. The only source of dust destruction present in this case, i.e. astration, does not seem to play a dominant role in determining the dust depletion pattern, once destruction by $\mathrm{SNe}$ and accretion in the ISM are neglected. We outline that neglecting these two processes is unrealistic since, as stressed in Sects. 2.2.2 and 2.2.3, there are robust proofs that destruction and accretion play a non negligible role in the evolution of dust in the S.N. (see McKee 1989; Draine 1990; Tielens 1998). For purpose of comparison, we also show the observational values derived by Kimura et al. (2003). The chemical composition of dust grains cannot be directly observed. The only way to estimate the abundance of an element locked up into dust is by means of its gas phase abundance, which is directly observable, and by means of an appropriate value representing the cosmic abundance. Observationally, for a given element $i$, the abundance in dust is derived by means of the subtraction between the cosmic abundance $\left(X_{i, \mathrm{C}}\right)_{\mathrm{obs}}$ and the observed gas abundances $\left(X_{\mathrm{gas}, i}\right)_{\mathrm{obs}}$ :

$\left(X_{i, \mathrm{dust}}\right)_{\mathrm{obs}}=\left(X_{i, \mathrm{C}}\right)_{\mathrm{obs}}-\left(X_{\mathrm{gas}, i}\right)_{\mathrm{obs}}$.

The observational determination of the dust fractions depends on the assumptions on the total cosmic abundances $\left(X_{i, \mathrm{C}}\right)_{\text {obs }}$ and on the $\mathrm{H}$ ionisation fractions $\chi_{\mathrm{H}}=0.25$. In the two panels of Fig. 3 , we consider four different sets of observed dust fractions (for details see caption of Fig. 3), calculated with various assumptions of both $\left(X_{i, \mathrm{C}}\right)_{\text {obs }}$ and $\chi_{\mathrm{H}}=0.25$.

In the upper panel of Fig. 3, the predicted values have been calculated by taking dust production into account in stars, dust destruction, and dust accretion in the ISM. The calculation of dust destruction and accretion depends on the choice of two parameters, i.e. the destruction efficiency $\epsilon$ and the accretion timescale $\tau_{0, i}$, respectively. In this case, the predictions have been calculated by assuming $\epsilon \sim 0.2$ and $\tau_{0, i}=5 \times 10^{7}$ yr for all elements. For this choice, the dust fractions are nearly independent of the choice of the dust condensation efficiencies. This confirms the results by Dwek (1998), who has shown that, at the present time, the accretion rate balances the destruction rate, and that the balance determines the depletion. Similar conclusions are drawn from the analysis of Zhukovska et al. (2007). By means of a different approach, Tielens (1998) attempted to determine the accretion and destruction rates from the observed depletions. The implication of these results is that, to derive constraints on the condensation efficiencies, one has to study the depletion pattern in systems where either destruction or accretion are absent. In Sect. 3.3.4, we show that systems suited to this study are the dwarf irregular galaxies, where dust accretion is likely to play a negligible role.

From the upper panel of Fig. 3, we also note that, for some elements, the assumption of a constant destruction efficiency provides a very poor fit to the observed dust fractions. These elements are $\mathrm{C}, \mathrm{O}$, and $\mathrm{S}$, for which the predictions indicate current dust fractions of $\sim 0.9$, higher than the values observed in the Local Interstellar Cloud.

The observed dust fractions can be reproduced in two ways by assuming that either the dust accretion timescale or the destruction efficiency depends on the physical properties of the chemical element. The dust accretion timescale is related to the micro-physics of the molecular clouds, which can not be modelled properly with our instruments. On the other hand, the destruction efficiency is connected to the heating of the ISM, 


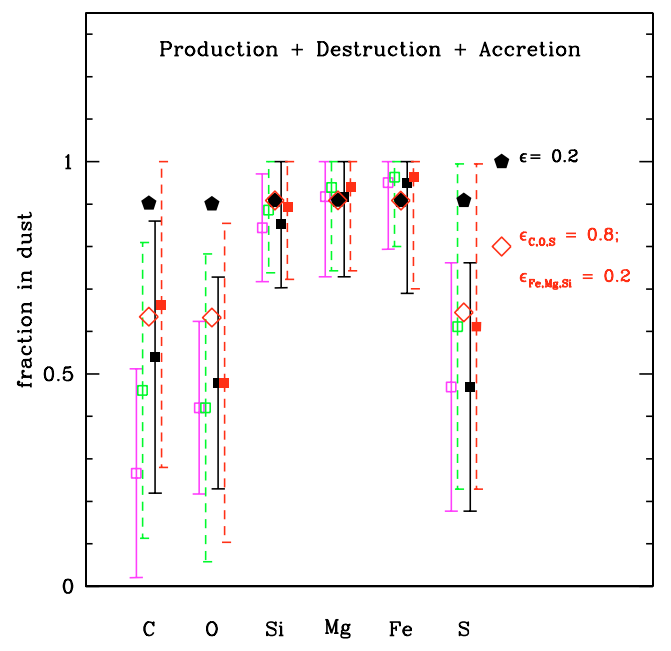

Fig. 4. Fractions in dust for various elements. The solid pentagons are the predicted present-day fractions calculated by assuming a dust destruction efficiency of $\epsilon=0.2$ for all elements. The open diamonds are the present-day fractions calculated assuming a dust destruction efficiency of $\epsilon_{\mathrm{C}, \mathrm{O}, \mathrm{S}}=0.8$ for $\mathrm{C}, \mathrm{O}$, and $\mathrm{S}$ and whereas for $\epsilon_{\mathrm{Fe}, \mathrm{Mg}, \mathrm{Si}}=0.2$ for $\mathrm{Fe}, \mathrm{Si}$, and $\mathrm{Mg}$. The squares with solid and dashed error bars are the same as in Fig. 3.

mainly due to $\mathrm{SNe}$ and their explosion rate, which is accounted for in detail by our models. In this paper, we assume that the dust destruction efficiency depends on the properties of the chemical element. A physical justification is related to the condensation temperatures of the various elements. These quantities express the volatility of the elements in dust (Lodders 2003, and references therein). In general, elements with higher condensation temperatures are more likely to aggregate into dust grains and are more resistant to destruction. Lodders (2003) has calculated the condensation temperatures for various elements for a solar-system composition gas. Her results indicate condensation temperatures of $78 \mathrm{~K}, 182 \mathrm{~K}$, and $704 \mathrm{~K}$ for $\mathrm{C}, \mathrm{O}$, and $\mathrm{S}$, respectively. On the other hand, the condensation temperatures calculated for $\mathrm{Fe}, \mathrm{Si}$, and $\mathrm{Mg}$ are $1357 \mathrm{~K}, 1529$, and 1397, respectively, hence considerably higher than for $\mathrm{C}, \mathrm{O}$, and $\mathrm{S}$. Here, we assume that the destruction efficiencies vary as a function of the condensation temperatures of the elements. For C, O, and $\mathrm{S}$ we assume a dust destruction efficiency of $\epsilon_{\mathrm{C}, \mathrm{O}, \mathrm{S}}=0.8$, whereas for $\mathrm{Fe}, \mathrm{Si}$, and $\mathrm{Mg}$ we assume $\epsilon_{\mathrm{Fe}, \mathrm{Mg}, \mathrm{Si}}=0.2$. As can be seen from Fig. 4, where we show the dust fractions calculated by assuming the above set of dust destruction efficiencies, with these values, it is possible to reproduce the observed dust fractions with good accuracy.

In Fig. 5, we show the time evolution of the dust fractions for various elements $(\mathrm{C}, \mathrm{O}, \mathrm{Fe}$, and $\mathrm{S})$. We have omitted the $\mathrm{Mg}$ and $\mathrm{Si}$ because for both elements, the evolution of the fraction in dust is identical to the one predicted for Fe. The curves for $\mathrm{C}$, O, and $\mathrm{S}$ show a similar behaviour after the minimum at $2 \mathrm{Gyr}$, with an increase up to the present time that is steeper than the one for Fe. This is due to the different destruction efficiencies adopted for the $\mathrm{C}, \mathrm{O}$, and $\mathrm{S}$ group and for the $\mathrm{Si}, \mathrm{Fe}$, and $\mathrm{Mg}$ group.

\subsubsection{The dust destruction and accretion rates}

Once we tuned the destruction efficiency $\epsilon$ and chosen a realistic value for the accretion timescale $\tau_{0, i}$, now we study the evolution of the accretion and destruction rates and compare our values to the ones estimated by other authors. In the left panel of

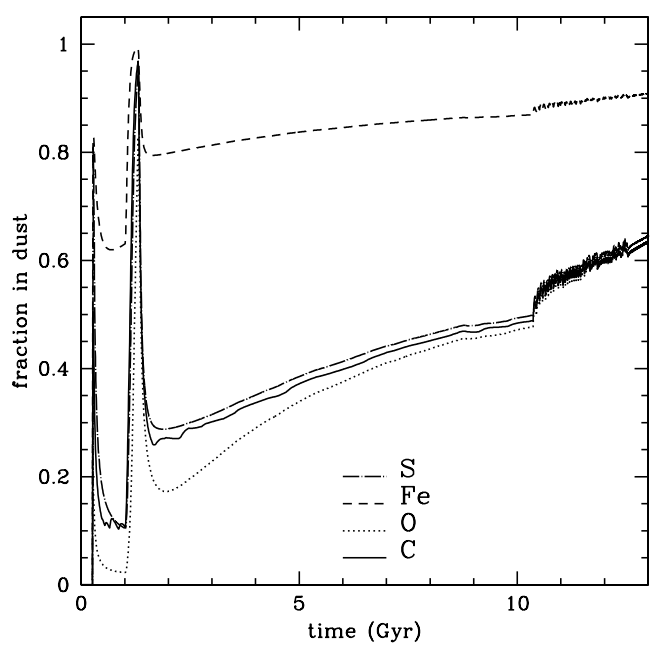

Fig. 5. Time evolution of the fractions in dust for various elements: $\mathrm{C}$ (solid line), O (dotted line), Fe (dashed line), and S (dot-dashed line). The evolution of the dust fractions for $\mathrm{Mg}$ and $\mathrm{Si}$ is identical to the Fe one.

Fig. 6, we show the predicted evolution of the dust destruction and accretion rates for the S.N. The evolution of both rates is sensitive to the SF and gas accretion history of the model for the S.N. In the literature, no observational estimate can be found for the accretion or destruction rates in the S.N. These quantities have been calculated by various authors on the basis of theoretical investigations (McKee 1989; Draine 1990; Jones et al. 1994; Tielens 1998). Our model is one-phase and represents one single medium dominated by cold, neutral gas. It is interesting to compare the rates predicted by our models with the average estimates by Draine (1990) and Tielens (1998) for a cold neutral medium. The values by Draine (1990) and Tielens (1998) are order-of-magnitude estimations and are provided with no error bar. For the dust destruction rate, we predict current values of $\sim 5 \times 10^{-9} \mathrm{yr}^{-1}$ and $\sim 8 \times 10^{-9} \mathrm{yr}^{-1}$ for silicate and carbon dust, respectively. Similar values are predicted for the accretion rates for both dust types. Our values are in good agreement with the order-of-magnitude estimations determined by Draine (1990) and Tielens (1998) with different approaches.

Several recent observational and theoretical results have addressed the role of $\mathrm{SNe}$ in dust production in the Milky Way (Dunne et al. 2003) and in high redshift galaxies (Hughes et al. 1998; Bertoldi et al. 2003; Morgan \& Edmunds 2003; Maiolino et al. 2004). Beside producing dust, $\mathrm{SNe}$ are the main sources of energy injection into the ISM, and their hot cavities are known to destroy dust grains (D98). Our aim here is to compare the type Ia and II SN destruction and production rates and see how do these quantities evolve with cosmic time. In the right panels of Fig. 6, we study the balance between production and destruction by $\mathrm{SNe}$ for C dust (lower panel) and $\mathrm{Si}$ dust (upper panel). For type II SNe, dust destruction dominates dust production throughout almost all of the cosmic history. Only for a very short time ( $\sim 0.3$ Gyr after the beginning of SF) was the type II SN production rate higher than the type II SN destruction rate. This agrees with the results by D98, who found that at earlier epochs, when the ISM metallicity was below $Z \sim 0.001$ (reached, according to our model, in $\sim 0.35 \mathrm{Gyr}$ ), SNe inject more dust into the ISM than they can destroy.

On the other hand, for type Ia SNe during the first Gyr of galactic evolution, Si dust production has dominated dust destruction, but with negligible consequences on the total Si dust 

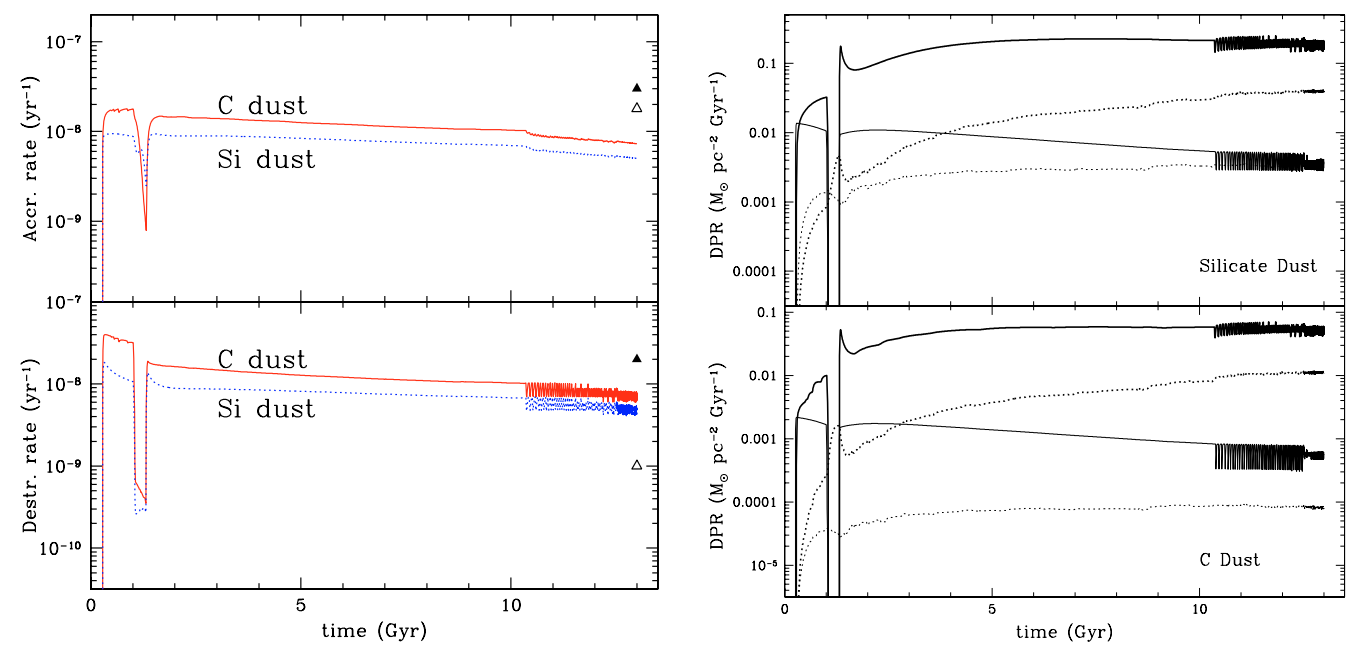

Fig. 6. Left: evolution of the dust accretion (upper panel) and destruction (lower panel) rates in the S.N. The solid and dotted lines represent the calculated rates for carbon and silicate dust, respectively. The open triangles and solid triangles are the present-day average values estimated by Draine (1990) and Tielens (1998), respectively, for a cold neutral medium. Right: evolution of the production and destruction rates by SNe for C dust (lower panel) and Si dust (upper panel). The thin dotted and solid lines are the predicted dust production rates by type Ia and type II SNe, respectively. The thick dotted and solid lines are the predicted dust destruction rates by type Ia and type II SNe, respectively.
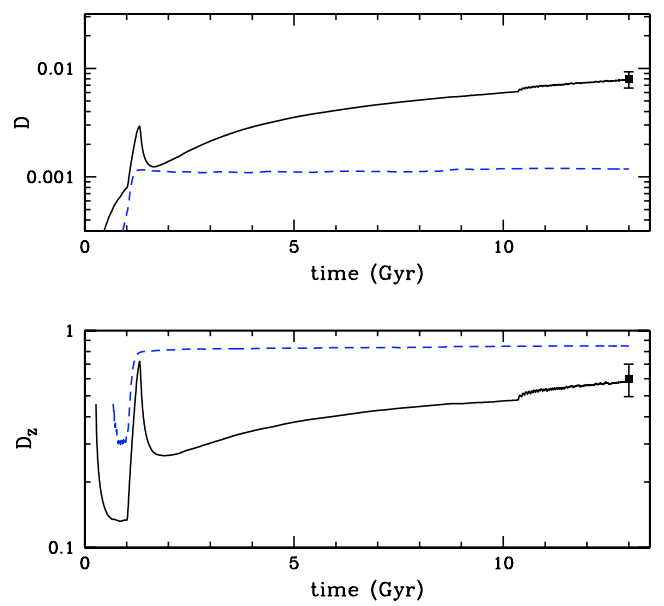

Fig. 7. Upper panel: predicted evolution of the dust-to-gas ratio in the S.N. (solid line) and at $16 \mathrm{kpc}$ from the Galactic centre (dashed line). The solid square with error bar is the observational value for the Galaxy taken from Issa et al. (1990). Lower panel: predicted evolution of the dust-to-metal ratio in the S.N. (solid line) and at $16 \mathrm{kpc}$ from the Galactic centre (dashed line). The open square with error bar is the observational value for the Galaxy assuming the dust-to-gas ratio estimated by Issa et al. (1990) and a solar metallicity of $Z_{\odot}=0.0133$ (Lodders 2003).

mass. We conclude that, according to our results, throughout the whole history of the S.N., the presence of SNe, in particular of type II SNe, is fundamental to the process of cycling the dust back into the ISM.

\subsubsection{Dust-to-gas and dust-to-metal ratios and their evolution}

We define the dust-to-gas ratio $D$ as the ratio between the dust mass and the total ISM mass surface densities as

$D=\frac{\sigma_{\text {dust }}}{\sigma_{\text {gas }}}$ while the dust-to-metal ratio $D_{Z}$ is the ratio between the dust mass and the metal mass surface density in the ISM,

$D_{Z}=\frac{\sigma_{\text {dust }}}{\sigma_{\text {gas }} \cdot Z}=\frac{D}{Z}$.

By studying the $D$ quantity in local spirals, Issa, MacLaren \& Wolfendale (1990) have found a correlation between $D$ and the metallicity. The implication of this fact is that the dust-to-metals ratio is nearly constant for local spirals. In the upper panel of Fig. 7, we show the predicted time evolution of D for two different zones of our Galaxy: the S.N. and a circular ring $1 \mathrm{kpc}$ wide located at a distance of $16 \mathrm{kpc}$ from the Galactic center. The latter represents a model for the outermost regions of the spiral disc (see Table 1). The comparison between these two models is interesting for understanding the differences of the dust properties for two regions located in different zones of the Galactic disc. We also plot the Galactic value $D_{\odot}=0.008$, taken from Issa et al. (1990), which is well-reproduced by our model.

In the lower panel of Fig. 7, we plot the time evolution of the dust-to-metal ratio $D_{z}$ for the S.N. and the spiral outskirts models. We compare the predictions to an observational value derived for the Galaxy. By assuming a metallicity of $Z_{\odot}=0.0133$ for the S.N. (Lodders 2003), we obtain $D_{Z, \odot}=0.6$ for our Galaxy. Interestingly, this value coincides with our predicted dust fraction for $\mathrm{O}$ (see Fig. 5), which is the element dominating the total metallicity.

Chemical evolution studies have shown that the dust-tometal ratio should not vary much during galaxy evolution (Inoue 2003; Edmunds 2001). Our predictions indicate that in the initial phases of galaxy evolution, in particular from the halo phase to the SF hiatus, $D_{z}$ experiences strong variations, evolving rapidly from 0.15 to 0.8 . After the peak at $1 \mathrm{Gyr}, D_{z}$ drops sharply and its evolution in the disc phase is much slower, i.e. a factor of $\sim 2$ from 2 Gyr up to the present time. This is consistent with the results by Inoue (2003). The present-day value is nicely reproduced by our models. The spiral outskirt model presents $D_{z}$ values higher than the S.N. model. Dust accretion is likely to take place in the outermost regions of the Milky Way owing to the presence of molecular clouds. In fact, recent near-infrared observations have revealed the presence of molecular clouds in 
Table 2. Comparison between some current output quantities predicted by means of our model for the solar neighbourhood, with the same quanties as computed by D98 and with the corresponding observational values (when available).

\begin{tabular}{llll}
\hline \hline Quantity & \multicolumn{3}{l}{ Our model D98 model Observed } \\
\hline ISM properties & 2.7 & 3.8 & $2-10^{a}$ \\
$\psi\left(R_{\odot}, T_{0}\right)\left(M_{\odot} \mathrm{pc}^{-2} \mathrm{Gyr}^{-1}\right)$ & 7 & 8.8 & $7-16^{b, c}$ \\
$\sigma_{\text {gas }}\left(R_{\odot}, T_{0}\right)\left(M_{\odot} p c^{-2}\right)$ & 1 & 0.8 & $0.3-1.5^{d}$ \\
$\dot{\sigma}_{\text {infall }}\left(R_{\odot}, T_{0}\right)\left(M_{\odot} \mathrm{pc}^{-2} \mathrm{Gyr}^{-1}\right)$ & & & \\
\hline Dust production in stars & & \\
$D P R_{\mathrm{C}, \mathrm{LIMS}}\left(R_{\odot}, T_{0}\right)\left(M_{\odot} \mathrm{pc}^{-2} \mathrm{Gyr}^{-1}\right)$ & $4 \times 10^{-4}$ & $2.8 \times 10^{-3}$ \\
$D P R_{\mathrm{C}, \mathrm{SN}}\left(R_{\odot}, T_{0}\right)\left(M_{\odot} \mathrm{pc}^{-2} \mathrm{Gyr}^{-1}\right)$ & $6 \times 10^{-4}$ & $1.6 \times 10^{-3}$ \\
$D P R_{\mathrm{Si}, \mathrm{LIMS}}\left(R_{\odot}, T_{0}\right)\left(M_{\odot} \mathrm{pc}^{-2} \mathrm{Gyr}^{-1}\right)$ & $1.4 \times 10^{-3}$ & $3.7 \times 10^{-3}$ \\
$D P R_{\mathrm{Si}, \mathrm{SN}}\left(R_{\odot}, T_{0}\right)\left(M_{\odot} \mathrm{pc}^{-2} \mathrm{Gyr}^{-1}\right)$ & $8 \times 10^{-3}$ & $1.1 \times 10^{-2}$ \\
\hline Dust accretion in the ISM & & \\
$D A R_{\mathrm{C}}\left(R_{\odot}, T_{0}\right)\left(M_{\odot} \mathrm{pc}^{-2} \mathrm{Gyr}^{-1}\right)$ & 0.065 & 0.035 \\
$D A R_{\mathrm{Si}}\left(R_{\odot}, T_{0}\right)\left(M_{\odot} \mathrm{pc}^{-2} \mathrm{Gyr}^{-1}\right)$ & 0.23 & 0.18 \\
\hline Dust destruction due to SN shocks & & \\
$D D R_{\mathrm{C}}\left(R_{\odot}, T_{0}\right)\left(M_{\odot} \mathrm{pc}^{-2} \mathrm{Gyr}^{-1}\right)$ & 0.058 & 0.033 \\
$D D R_{\mathrm{Si}}\left(R_{\odot}, T_{0}\right)\left(M_{\odot} \mathrm{pc}^{-2} \mathrm{Gyr}^{-1}\right)$ & 0.25 & 0.19 \\
\hline$D_{\mathrm{C}}$ & $8.5 \times 10^{-4}$ & $1.7 \times 10^{-3}$ \\
$D_{\mathrm{Si}}$ & $4.8 \times 10^{-3}$ & $7 \times 10^{-3}$ \\
\hline
\end{tabular}

Note: $R_{\odot}=8 \mathrm{kpc}$ is the solar distance from the Galactic center. References: ${ }^{a}$ Rana (1991). ${ }^{b}$ Dickey (1993). ${ }^{c}$ Kulkarni \& Heiles (1987). ${ }^{d}$ Portinari et al. (1998).

regions at galactocentric distances out to $R \geq 13.5-20 \mathrm{kpc}$ (Kobayashi \& Tokunaga 2000; Snell et al. 2002; Nakagawa et al. 2005).

In Table 2, we present a detailed comparison between the numeric values as predicted by means of our model for the S.N. with the predictions by D98, as well as with the available corresponding observational results. Our results for the predicted ISM properties (second column) are very similar to the ones of D98 (third column) and well within the available observational values (fourth column). The small differences are due mainly to different prescriptions for the SF law (see our Eqs. (5) and (15) of D98) and for the infall law, for which we assume the two-infall expression by Chiappini et al. (1997), whereas D98 assumes a simple exponential law.

Differences in the $\mathrm{C}$ and $\mathrm{Si}$ dust production rates from LIMS and $\mathrm{SNe}(\mathrm{Ia}+\mathrm{II})$ are mainly due to the different nucleosynthetic prescriptions used in this paper and by D98. In particular, for stars of low and intermediate mass we use the metallicitydependent yields by Van den Hoeck \& Groenewegen (1997), whereas, for stars in the same mass range, D98 uses the yields computed by Renzini \& Voli (1981). For the dust production rates, we decided in Table 2 not to show any observational value since the only existing observational constraints are the ones by Jones \& Tielens (1994), which are provided without any error bar.

Differences in the dust accretion rates $(D A R)$ are due to the different present-day gas densities and to the different nucleosynthesis prescriptions, whereas the small differences in the destruction rates $(D D R)$ are due to the different star formation laws, which cause differences in the predicted SNRs. Finally, in the last two lines of Table 2, we compare the $\mathrm{C}$ and Si dust to gas ratios as computed by us for the time when the solar system formed (i.e. at $9.5 \mathrm{Gyr}$ ), with the values as predicted by D98.

\subsection{Dust evolution in elliptical galaxies}

In this section we make use of the most accurate values for the parameters derived above for the S.N., in order to extend our formalism to the class of early-type galaxies, implementing Eq. (7) in the model by Pipino et al. (2005).

As a first step, we do not modify the parameters related to processes such as the dust growth, which are mainly governed by the microphysics, so they should not depend on the galactic morphology. However, after the onset of the galactic wind, ellipticals are basically devoid of cold gas. Therefore, we assume that, during this phase, no dust accretion can occur in elliptical galaxies.

Concerning the last term in Eq. (7), dust is now allowed to escape the ISM during the galactic wind, although no conclusion can be drawn on its fate. Therefore, the dust loss rate in Eq. (7) switches on during the galactic wind, and it has been assumed that it follows the gas flow rate. In particular, we recall that Pipino et al. (2002, 2005) successfully reproduced the amount of metals injected into the intra cluster medium (ICM) by assuming that, at each timestep, the ejected mass $\Delta M$ was set by the following condition: $\frac{\Delta M}{M_{\mathrm{gas}}}=\frac{\Delta E}{E_{\mathrm{th}}}$, where $\Delta E$ was the difference between the thermal energy $E_{\text {th }}$ and the gas binding energy. Here we assume a similar condition to also hold for the dust flow rate. Therefore, we find that the fraction of the ISM (i.e. dust plus gas) that can escape scales with $\Delta E$ as

$$
\frac{\Delta M}{M_{\mathrm{ISM}}}=\frac{\Delta M_{\mathrm{dust}}+\Delta M_{\mathrm{gas}}}{M_{\mathrm{dust}}+M_{\mathrm{gas}}}=\frac{\Delta E}{E_{\mathrm{th}}} .
$$

We are aware that the infrared emission from grains might change the cooling function (Draine 1981). To tackle this issue, it is necessary to study interstellar dust from a spectrophotometric point of view. This will be the subject of a forthcoming paper.

\subsubsection{The dust production rates}

In Fig. 8, we show the predicted dust production rates for the La1 model, describing an elliptical galaxy of stellar mass $\sim 10^{11} M_{\odot}$. During the starburst phase, lasting $\sim 1 \mathrm{Gyr}$ in this case, the major $\mathrm{C}$ and $\mathrm{Si}$ dust producers are type II SNe. After the end of the starburst, Si dust production is dominated by type Ia $\mathrm{SNe}$, whereas $\mathrm{C}$ dust production is mainly due to type Ia SNe. At late times, i.e. after 10 Gyr, LIMS are the major $\mathrm{C}$ dust producers.

\subsubsection{The evolution of the dust fractions}

In Fig. 9, we show the predicted evolution of the dust fractions locked up in $\mathrm{C}, \mathrm{O}, \mathrm{S}$, and Fe for the fiducial case Lal+Itoh. In the very early phase of the evolution (i.e. $t \leq 200 \mathrm{Myr}$, when the gas is still infalling and the SFR has just reached its maximum intensity), we predict that nearly $80 \%$ of $\mathrm{Si}$ and $\mathrm{Fe}$ are locked into dust, whereas only $40 \%$ of $\mathrm{C}$ and $10 \%$ of $\mathrm{O}$ are so. This finding roughly agrees with the depletion pattern found for spirals. After an initial peak, the dust fractions undergo a sudden decrease due to the high SN activity, which leads to high destruction rates. The decrease in the dust fractions is then enhanced by the mass removal as soon as the galactic wind develops. After the end of the galactic wind (around $7 \mathrm{Gyr}$ ), the dust mass can increase again, before the sputtering due to the hot X-ray emitting gas can play a major role in the grain destruction. An interesting case is represented by the $\mathrm{S}$ dust fraction. In spirals, the evolution of the 


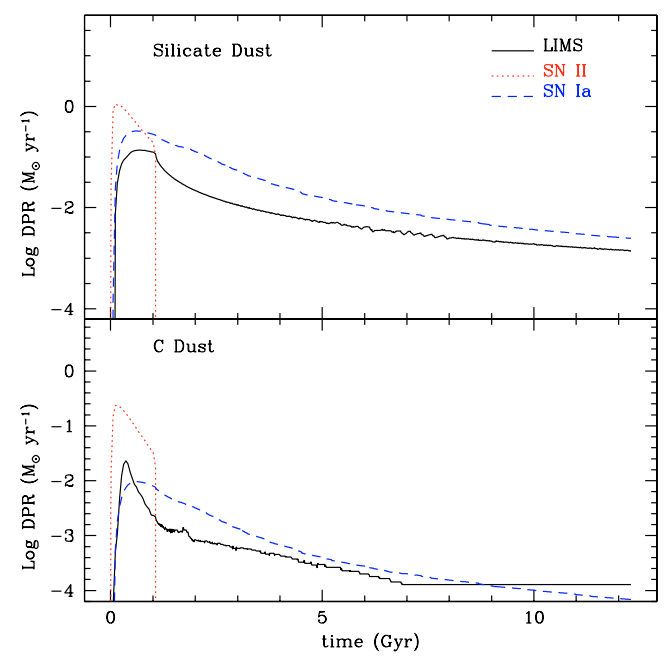

Fig. 8. Predicted dust production rates from various sources for the chemical evolution model La1+Itoh (fiducial case) for an elliptical galaxy. In the lower (upper) panel, we show the results for the carbon (silicate) dust. Solid lines: contribution by low and intermediate mass stars (LIMS). Dotted lines: contribution by type II SNe. Dashed lines: contribution by type Ia SNe.

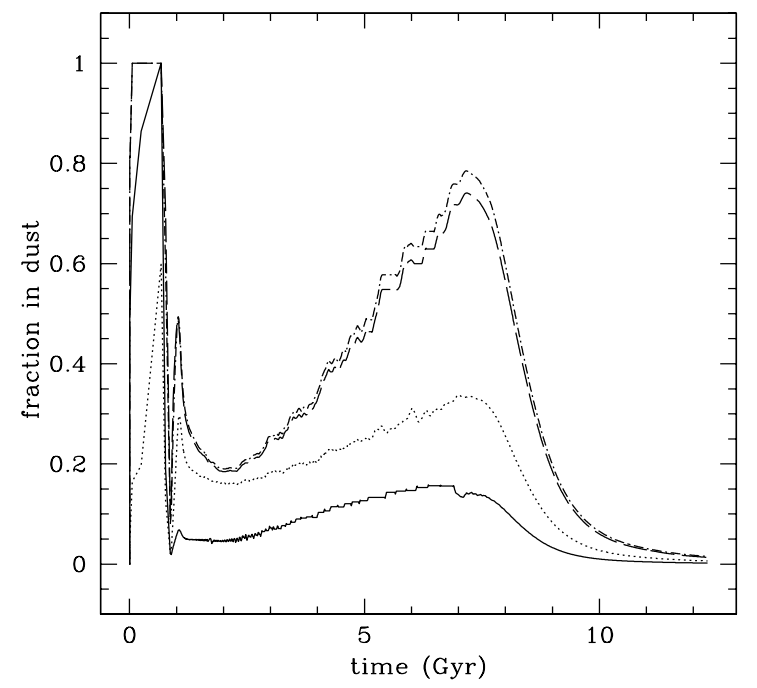

Fig. 9. Fractions locked in dust for C (solid line), O (dotted), S (dotdashed), $\mathrm{Fe}$ (dashed) for fiducial models Ha1 (left panel), and La1 (right panel).

$\mathrm{S}$ dust fraction is similar to the ones of $\mathrm{C}$ and $\mathrm{O}$, mainly because of the similar destruction efficiencies. In these systems, the destruction is very efficient because of the continuous explosions of type II SNe. On the other hand, dust destruction plays a minor role in ellipticals, presenting long periods of no star formation activity. In these systems, the evolution of the $\mathrm{S}$ dust fraction is close to the one of $\mathrm{Fe}$, since the two elements have similar condensation efficiencies.

\subsubsection{The dust fractions in present-day ellipticals: local ellipticals and the Fe discrepancy}

The main answer that we expect from our analysis is whether the inclusion of the dust treatment may help in solving the so-called iron discrepancy (Arimoto et al. 1997) in X-ray spectra of the hot halos surrounding ellipticals. When the ASCA satellite provided the first reliable measure of the iron abundance in the hot ISM of ellipticals (e.g. Awaki et al. 1994; Matsumoto et al. 1997), it was much lower than the solar value, at odds not only with theoretical models for elliptical galaxies available at that time (Arimoto \& Yoshii 1987; Matteucci \& Tornambé 1987), which predicted that their ISM should exhibit $[\mathrm{Fe} / \mathrm{H}]>0$, but also with the mean metallicity of the stellar component inferred from optical spectra. This issue then had been partly alleviated by taking temperature gradients into account (Buote \& Fabian 1998; Buote 1999). Recently, Humphrey \& Buote (2006) reported emission-weighted $\mathrm{Fe}$ abundances up to $\sim 2-3$ solar in a sample of 28 early-type galaxies, corresponding to $[\mathrm{Fe} / \mathrm{H}]=0.3-0.48$. Nevertheless, the iron discrepancy persists when comparing the recent results by Pipino et al. (2005), predicting $[\mathrm{Fe} / \mathrm{H}] \geq 0.85$ in the ISM of elliptical galaxies, to the most recent abundance measurements in the X-ray spectra.

The Fe condensation in dust is among the possible physical mechanisms often invoked to solve this issue (see Arimoto et al. 1997, for a comprehensive analysis). Recent far-infrared observations, in fact, claim that the dust mass in ellipticals could be $\sim 10^{6-7} M_{\odot}$, higher than previous estimates (Temi et al. 2004) by a factor of ten, with $\sim 2 \times 10^{5} M_{\odot}$ of the dust residing in the very central galactic regions (Leeuw et al. 2004). Independent, recent observations carried out by means of the SCUBA camera on local ellipticals (Vlahakis et al. 2005) seem to confirm these values, although the sample should be enlarged and the effects of synchrotron radiation clarified. Recently, several observationalal determinations of the dust masses in ellipticals have been possible thanks to the Spitzer Space Telescope. In elliptical galaxies, Kaneda et al. (2007) derive dust masses typically about $10^{5}-10^{6} M_{\odot}$. In an early type galaxy of the Virgo group, Panuzzo et al. (2007) obtain a dust mass of $1.2 \times 10^{6} M_{\odot}$. These new observational values are well reproduced by our fiducial models, which predict a dust mass of $\sim 1.1 \times 10^{6} M_{\odot}$ for more than 10 Gyr old giant ellipticals $\left(\sim 0.8 \times 10^{6} M_{\odot}\right.$ of dust for a more typical galaxy like our model La1).

The predicted evolution of the Fe dust destruction rates and of the Fe fraction in dust for the Lal model assuming the Itoh and the McKee prescriptions for the destruction are presented in the upper and lower panels of Fig. 10, respectively. In Fig. 11, we show the predicted evolution of the Fe abundance in the hot phase of a typical elliptical galaxy by adopting the Itoh (1989) and the Mckee (1989) prescriptions. For the fiducial model, the predicted present-day iron abundance in the hot gas is $[\mathrm{Fe} / \mathrm{H}]=+0.6$ dex (for both La1 and Ha1). These abundances are a factor of 2-5 lower than the values predicted by the original Pipino et al. (2005) models La1 and Ha1, respectively. Several factors act in determining this result: dust depletion (up to $20 \%$ of $\mathrm{Fe}$ is in dust at late times), a more prolonged wind phase with respect to the original model Ha1 (which leads to a more efficient Fe ejection), or different stellar yields. Moreover, since the Fe emission line dominates the X-ray spectrum of the hot ISM, a reduced $\mathrm{Fe}$ abundance in the gas leads to better agreement between the predicted X-ray luminosity $\left(2.1 \times 10^{42} \mathrm{erg} \mathrm{s}^{-1}\right)$ and temperature $(\sim 0.7 \mathrm{kev})$ for the hot halo of giant ellipticals with the observed values than in Pipino et al. (2005) case. When adopting the $L a l+M K$ prescription (in Figs. 12 and 10), the grain destruction is considerably reduced and up to $\sim 4 \times 10^{8} M_{\odot}$ of the dust can survive after 12 Gyr of galactic evolution. At this stage, the amount of Fe locked up in dust is close to $60 \%$ (for a comparison, the fraction for $\mathrm{O}$ and $\mathrm{C}$ are only $25 \%$ and $12 \%$, respectively), leading to a $[\mathrm{Fe} / \mathrm{H}]=0.15$ (Ha1) -0.3 (La1) dex, as well as $[\mathrm{O} / \mathrm{Fe}]=-0.12 \mathrm{dex}$, in excellent agreement with the available data (see Pipino et al. 2005, Table 4 for a compilation of the most recent ones): this might be the solution of the iron 

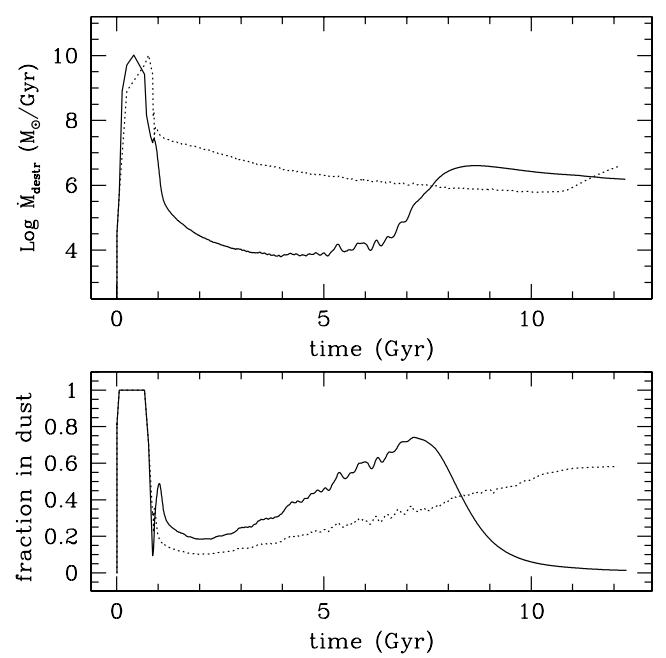

Fig. 10. Upper panel: temporal behaviour of the Fe grain destruction rates for an elliptical galaxy (Pipino et al. 2005, model La1). Dotted: $L a 1+M K$ case. Solid: Lal+Itoh case. Lower panel: evolution of the Fe dust fraction in the ISM of the above models.



Fig. 11. Evolution of the $\mathrm{Fe}$ abundance in the hot phase of an elliptical galaxy for the La1 model. Solid lines: La1+Itoh model. Dotted line: La1+MK model.

discrepancy. In contrast to other authors (Arimoto et al. 1997), we suggest that the iron discrepancy might be solved by taking the dust condensation of Fe into account. New and ellipticaldedicated calculations of the sputtering by hot (i.e. $T>10^{7} \mathrm{~K}$ ) plasmas are needed to eventually assess this issue.

\subsubsection{The dust flow into the IGM/ICM}

The amount of dust ejected by a giant elliptical galaxy can be obtained by integrating Eq. (18) over time. The amount is $\sim 3 \times 10^{9} M_{\odot}$ for the fiducial Ha1 model. For model La1 (fiducial case), instead, the ejected dust mass is $\sim 5 \times 10^{8} M_{\odot}$. Once the dust grains are ejected into the IGM, no firm conclusions can be drawn about their consequent evolution. For instance, we need a detailed comparison of the efficiency of dust removal by means of the $\mathrm{SN}$-driven wind with the dust destruction rate due to the $\mathrm{SN}$ explosions. Moreover, a careful treatment of the dust survivability in a medium such as the hot ICM/IGM is beyond the scope of the paper.
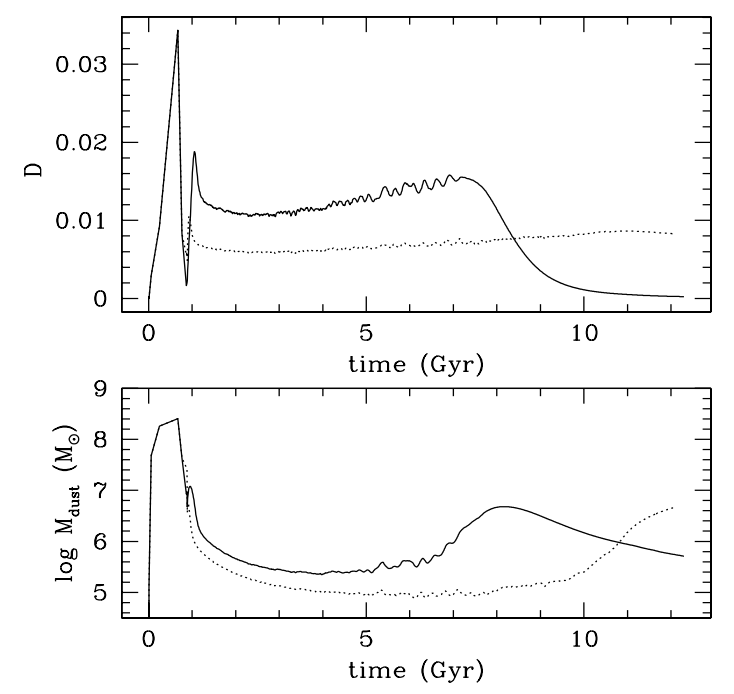

Fig. 12. Upper panels: dust to gas mass ratio $(D)$ for Pipino et al. (2005)'s model La1. Dotted: Lal $+M K$ case. Solid: Lal+Itoh case. Lower panel: evolution of the dust mass in the ISM for the above models.

\subsubsection{The dust fractions in young ellipticals: hints from Lyman Break and SCUBA galaxies}

Unfortunately, the are no direct measurement of the dust composition in young ellipticals available to us. Therefore, in this section, we will try to constrain our models by means of those objects that are believed to be their high-redshift counterparts.

It has been argued that the so-called SCUBA sources may be ellipticals in the process of formation (Lilly et al. 1999; Eales et al. 2000). The inferred SFRs of $\sim 100-1000 M_{\odot} / \mathrm{yr}$ are comparable to those assumed by our models, as well as the estimated duration of the formation process and stellar mass involved. A useful measure of the dust mass in such high redshift objects is given by Dunne et al. (2003). They fitted the dust mass function by means of a Schechter curve and found that the characteristic dust mass at the break is $M_{\mathrm{d}}^{*} \sim 4.7 \times 10^{8} M_{\odot}$ at redshift $z=5$. Similar values can be achieved by models such as Lal during their first Gyr of evolution (we expect these galaxies to be close to a typical present-day $L^{*}$ galaxy, Fig. 12) and are smaller than the ones predicted for the Ha1 model that should in turn represent the bright end of the present-day luminosity function.

Another class of objects that can trace the evolution of lowand intermediate-sized ellipticals are Lyman Break Galaxies (LBGs), which are starburst galaxies observed at high redshift $(z>2.5)$ and are identified by the colours of their far ultraviolet spectral energy distribution around the $912 \AA$ Lyman continuum discontinuity (Giavalisco 2002). The interpretation of their abundance patterns suggests that LBGs may represent young spheroids, observed during their main SF episode (Matteucci \& Pipino 2002, where we refer the reader interested in a more complete analysis), under the assumption that half of Fe was hidden in dust. Here we briefly repeat the study of Matteucci \& Pipino (2002) by comparing the output of the chemical evolution code with the observed abundances in MS1512-cB58, the brightest LBG known so far owing to its gravitationally lensed nature. A stellar mass of $\sim 10^{10} M_{\odot}$, an age lower than $\sim 300 \mathrm{Myr}$ and an SFR of $40 M_{\odot} \mathrm{yr}^{-1}$ (but we can consider a range of possible values of $20-80 M_{\odot} \mathrm{yr}^{-1}$ given the uncertainties affecting the derivation of the SFR), have been reported for MS1512-cB58 (Pettini et al. 2002). In order to have a realistic model for such a galaxy, we extend the class of models of Pipino et al. (2005) 

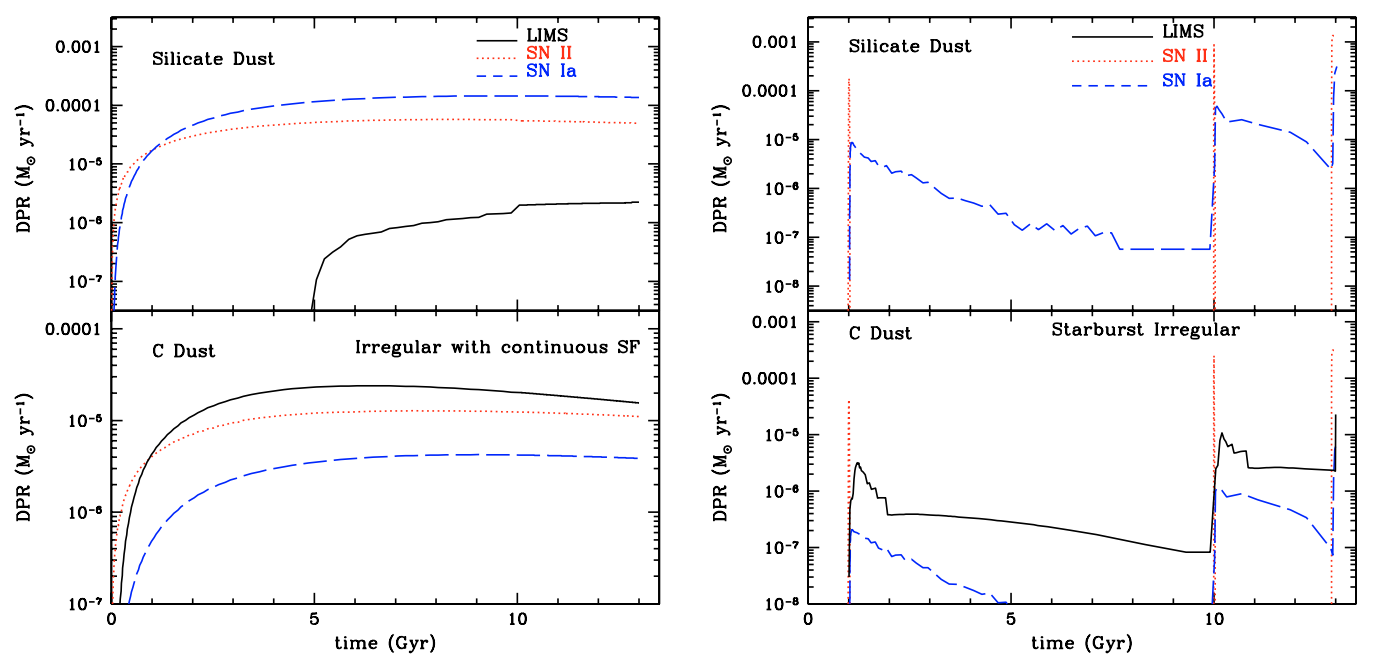

Fig. 13. Predicted dust production rates from various sources for a chemical evolution model of an irregular galaxy with continuous SF (left) and a starburst irregular galaxy (right). In the lower (upper) panels, we show the results for the carbon (silicate) dust. Solid lines: contribution by low and intermediate mass stars (LIMS). Dotted lines: contribution by type II SNe. Dashed lines: contribution by type Ia SNe.

down to a $M_{\text {lum }}=10^{10} M_{\odot}$ spheroid, with an SF efficiency $v=5 \mathrm{Gyr}^{-1}$ and an infall timescale $\tau=0.5 \mathrm{Gyr}$. The maximum value for the SFR is $\sim 20 M_{\odot} \mathrm{yr}^{-1}$. Since we focus on the very early phases of evolution, the assumptions regarding the dust destruction in the hot halos discussed in the previous paragraphs do not affect the results. At $130 \mathrm{Myr}$, our model predicts the following abundance ratios: $[\mathrm{Fe} / \mathrm{H}]=-1.13,[\mathrm{O} / \mathrm{H}]=-0.24$, $[\mathrm{Mg} / \mathrm{H}]=-0.62$, and $[\mathrm{Si} / \mathrm{H}]=-0.64$, in remarkable agreement with the values reported by Pettini et al. (2001) and Tepliz et al. (2000). In particular, they find $[\mathrm{Fe} / \mathrm{H}]=-1.10,[\mathrm{O} / \mathrm{H}]=-0.26$, as well as $[\mathrm{Mg} / \mathrm{H}],[\mathrm{Si} / \mathrm{H}] \sim-0.42$, with quoted uncertainties of about 0.2 dex. Pettini et al. (2002) claim that part (roughly half) of the $\mathrm{Fe}$ could be locked into dust. On the other hand, the theoretical expectation (based on the S.N.) that $\mathrm{Mg}$ and $\mathrm{Si}$ should also be depleted, although to a lesser extent, relative to $\mathrm{S}$ was not satisfied by their data. An answer can be given by our model, which in fact predicts that at $\sim 100 \mathrm{Myr}$ the dust fractions are $50 \%, 37 \%, 32 \%$ and $7 \%$ for $\mathrm{Fe}, \mathrm{Mg}$, S, and $\mathrm{O}$, respectively. Moreover, by means of our model, we can a posteriori prove the hypothesis made by Matteucci \& Pipino (2002) for the Fe depletion was right. The only difference between their model and this one is in the age estimate. In particular, we suggest an age of $\sim 100$ Myr here, whereas the previous estimate was close to 30 Myr. A slightly older age can be understood in terms of the formation process. The model used by Matteucci \& Pipino (2002) tightly followed the monolithic scenario, so the SFR was maximum at the beginning. The present model, owing to a nonnegligible infall (e-folding time of $0.5 \mathrm{Gyr}$ ), takes a longer period to assemble the same amount of stars.

For a more general comparison with LBGs, we find that the value for the dust-to-gas ratio $D=0.09 D_{\odot}$ predicted by this last model is in the range of values predicted by Inoue (2003). Moreover, we estimate a value of 0.13 (i.e. 0.2 times the S.N. value) for the quantity $D_{Z}$, which is consistent with the range $0.01-1 D_{Z, \odot}$, as predicted by Inoue (2003). At the same age, model La1 features $D_{Z} \sim 0.7 \geq D_{Z, \odot}$, and this value might reflect the fact that models such as Lal begin to be too massive to properly represent LBGs. These results reinforce the analysis by Matteucci \& Pipino (2002); therefore, we conclude that LBGs are very likely to be the progenitors of present-day low-mass spheroids.

\subsection{Dust evolution in irregular galaxies}

\subsubsection{Dust production rates}

In Fig. 13, we show the dust production rates for the models of an irregular galaxy with continuous SF and a starburst irregular. All the dust production rates calculated for the IC model have a smooth behaviour and in general present a continuous increase, lasting several Gyrs, and then reach a plateau. On the other hand, the production rates calculated for the starburst irregular reflect the shape of the SF and present a gasping or intermittent behaviour. An interesting difference between the dust production rates predicted for irregular galaxies and the one predicted for the S.N. model concerns the production of silicate dust by low and intermediate mass stars. In the case of the S.N. model, the Si dust production by LIMS starts immediately after the first SF episode. On the other hand, Si dust production by LIMS starts at $\sim 5$ Gyr for the IC model, whereas for the starburst model it does not start at all. This reflects that in dwarf galaxies and in low metallicity systems in general, it is rare to have $X_{\mathrm{C}} / X_{\mathrm{O}}>1$ (see Sect. 2.1).

\subsubsection{The dust fractions}

In Fig. 14, we show the predicted evolution of the dust fractions for the IC and the starburst irregular model. In the case of the IC model (Fig. 14, lower panel), the dust fractions are maximum at the beginning and decrease progressively up to the present time, when $\mathrm{C}, \mathrm{O}$, and $\mathrm{S}$ have dust fractions lower than $\sim 0.1$, whereas $\mathrm{Fe}$ is $\sim 0.3$. These values are considerably lower than the ones predicted for the S.N. and for the present-day ellipticals. In this case, the main reason is the absence of the dust accretion term and the continuous SF history, which gives rise to continuous type II SN explosions. As seen in Sect. 3.1.4, these objects are the main elements responsibles for dust destruction, so they play a dominant role in keeping the dust fractions low in irregular galaxies.

In the upper panel of Fig. 14, we show the evolution of the dust fractions for the starburst irregular model. This model is characterised by long periods of no SF activity. During these periods, all the dust fractions tend to remain constant or to increase, since the only contributors to destruction are type Ia SNe. Each 


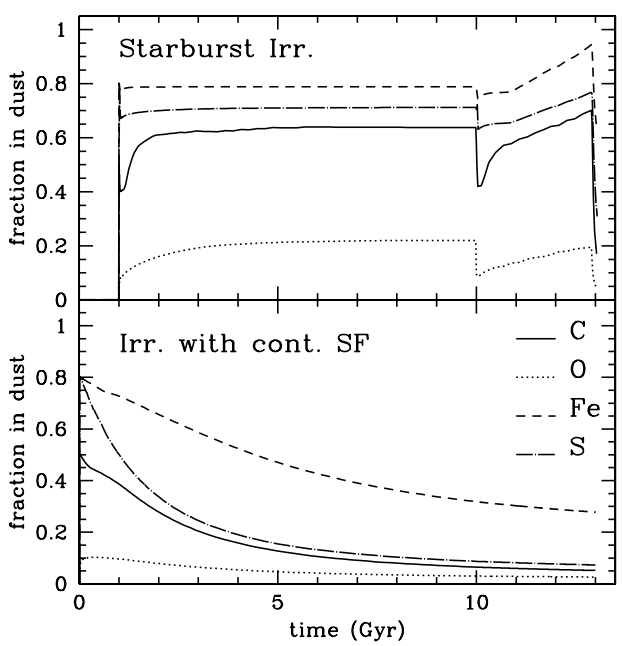

Fig. 14. Time evolution of the fractions in dust for various elements for the IC model (lower panel) and for the starburst model (upper panel). The solid lines, dotted lines, dashed lines, and dot-dashed lines represent the evolution of the $\mathrm{C}, \mathrm{O}, \mathrm{Fe}$, and $\mathrm{S}$ dust fractions, respectively. The evolution of the dust fractions for $\mathrm{Mg}$ and $\mathrm{Si}$ is identical to the $\mathrm{Fe}$ one.

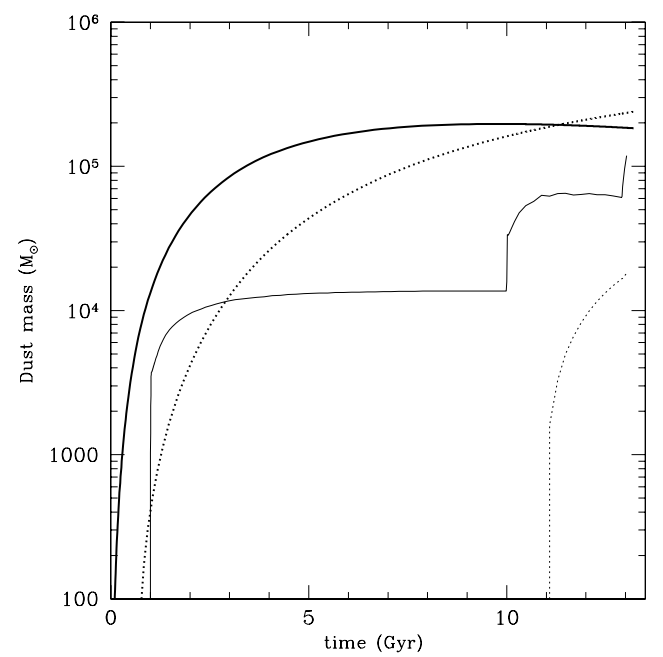

Fig. 15. Time evolution of the dust masses for the IC (thick lines) and starburst irregular (thin lines) models. Solid lines: dust mass locked up into the galaxies. Dotted lines: the dust masses ejected into the IGM.

starburst corresponds to massive type II SN activity, hence to considerable dust destruction and consequently sharp decrease of all the dust fractions, which is visible at 1, 10, and 13 Gyr. At the present time, the predicted dust fraction for the starburst model are, in general, higher than the ones calculated for the IC model.

\subsubsection{Dust masses locked up in galaxies and ejected into the IGM}

In Fig. 15, we show the predicted evolution of the dust masses for IC and starburst models, with the dust masses locked up into the galaxies and the dust masses ejected into the IGM. Our predictions indicate that, for an irregular galaxy of $\sim 5 \times 10^{8} M_{\odot}$, the present-day galactic dust masses are of the order of $1-2 \times$ $10^{5} M_{\odot}$, irrespective of the SF history. These values are compatible with the dust masses observed at the present time in dwarf irregulars (dIrr) and blue compact galaxies (BCG), which can span from $\sim 10^{2} M_{\odot}$ up to $\sim 10^{6} M_{\odot}$ (Lisenfeld \& Ferrara 1998).
Our values for the dust masses in local dwarfs and irregulars are compatible also with observational determinations achieved by means of the Spitzer Space Telescope. Cannon et al. (2006) derive a total dust mass of $8.3 \times 10^{4} M_{\odot}$ for the local dwarf NGC 6822. For dwarf galaxies of the M 81 group, Walter et al. (2007) find dust masses of $10^{4}-10^{6} M_{\odot}$, in perfect agreement with the values we predict for dwarfs.

Larger differences are predicted for the dust masses ejected by IC and starburst irregular galaxies into the IGM. Our results indicate that, for the IC model, the galactic wind develops very early, at ages comparable to $\sim 1$ Gyr, whereas the onset of the wind occurs after $10 \mathrm{Gyr}$ of evolution for the starbust irregular. IC and starburst irregulars eject into the IGM $\sim 2 \times 10^{5} M_{\odot}$ and $\sim 1 \times 10^{4} M_{\odot}$ of dust, respectively. Calura \& Matteucci (2006a) have shown that the bulk of the IGM metal enrichment is due to giant spheroids, despite the fact that the number density of dwarf irregulars is higher than the one of the giant ellipticals. Since the ejected dust mass is proportional to the total ejected metal mass, we can conclude that ellipticals should be considered as the major contributors to the dust enrichment of the IGM.

\subsubsection{Dust-to-gas and dust-to-metals ratios}

In the case of irregular systems, the observed dust-to-gas ratio is generally defined as:

$D^{\prime}=\frac{M_{\mathrm{dust}}}{M_{\mathrm{HI}}}$

where $M_{\text {dust }}$ and $M_{\mathrm{HI}}$ are the dust and HI mass determined from the far infrared and from the radio emission, respectively (Lisenfeld \& Ferrara 1998). In the models, the neutral $\mathrm{H}$ mass is $M_{\mathrm{HI}}=M_{\mathrm{gas}} \cdot X_{\mathrm{H}}$, where $M_{\mathrm{gas}}$ is the total gas mass and $X_{\mathrm{H}}$ is the $\mathrm{H}$ mass fraction. In Fig. 16, we show the $D^{\prime}$ calculated for the IC model and for the starburst model as a function of time (upper left panel) and as a function of the metallicity (lower left panel), and compared to a set of observations in local dwarf galaxies. At any time, the IC model is characterised by higher $D^{\prime}$ values than the starburst model.

In the lower panel of Fig. 16, we use the quantity $12+$ $\log (\mathrm{O} / \mathrm{H})$ as a proxy of the metallicity. The observational values here are taken from a compilation by Lisenfeld \& Ferrara (1998) (see caption of Fig. 16), and refer to BCD and dIrr galaxies. The dust-to-gas ratio of dwarf irregulars and BCDs as a function of the metallicity has been studied by Hirashita (1999) and Hirashita et al. (2002). In particular, Hirashita et al. (2002) suggests that an intermittent SF history allows one to reproduce the observed dispersion in the relation between the dust-to-gas ratio and the metallicity.

The starburst model has a present-day metallicity lower than the IC model. We note that the predicted present-day values are in general higher than the majority of the observed values. The compilation of observations presented by Lisenfeld \& Ferrara (1998) is based on IRAS data. The observed determinations of the dust mass are likely to represent lower limits to the actual values, owing to the undetected cold dust component (D98, Popescu et al. 2002). Lisenfeld \& Ferrara (1998) estimate a total error in the dust to gas ratio to be of a factor of 4 , taking into account all the possible factors of uncertainty (contribution from very small grains, cold dust, molecular gas and variations in the $\mathrm{HI}$ and optical diameter). By taking this uncertainty into account, the observed dust-to-gas ratios are consistent with our predictions 

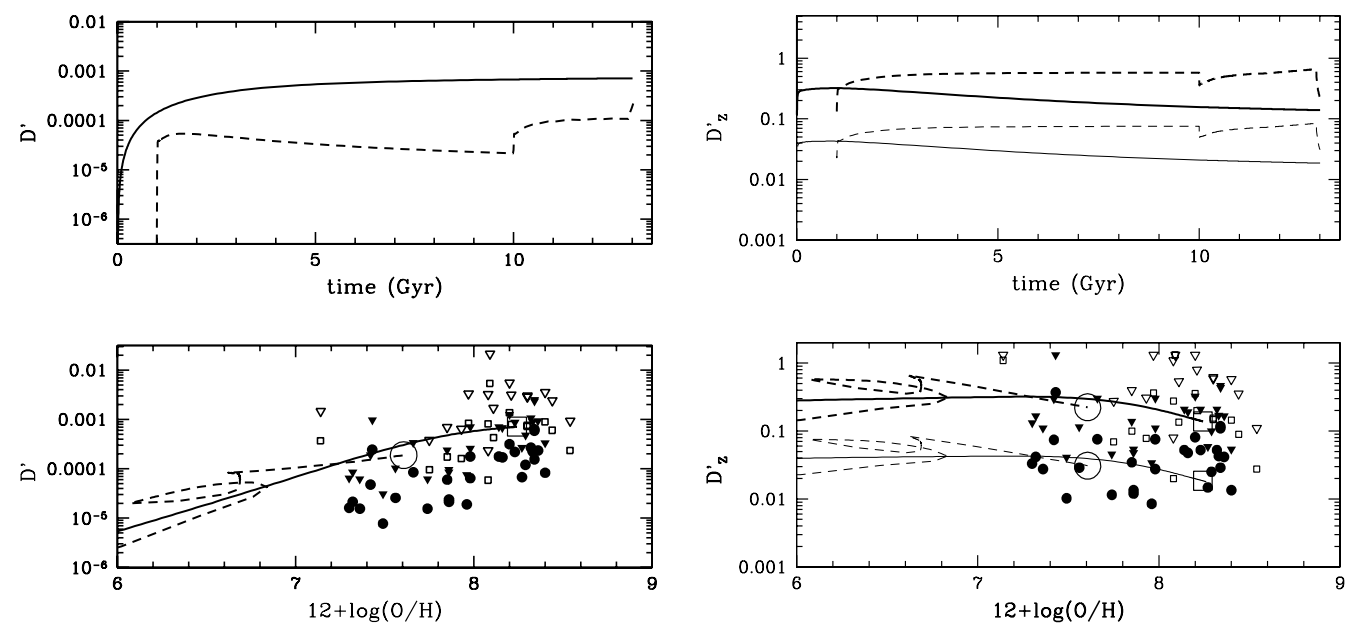

Fig. 16. Left: evolution of the dust to gas ratio calculated for the IC model (solid lines) and for the starburst model (dashed lines) as a function of time (upper panel) and metallicity (lower panel). In the lower panel, the solid circles and open squares are $D^{\prime}$ values observed in local dIrr and BCD galaxies, respectively (Lisenfeld \& Ferrara 1998). The solid and empty inverted triangles represent upper limits to the observations for local dIrr and BCD galaxies, respectively, calculated by assuming an uncertainty of a factor of 4. Right: evolution of the dust to metals ratio calculated for the IC model (solid lines) and for the starburst model (dashed lines) as a function of time (upper panel) and metallicity (lower panel). The thick and thin lines represent the predicted $D^{\prime}$ calculated assuming for the condensation efficiencies the values suggested by D98 and a constant value of 0.1 , respectively. All the symbols are as in the left lower panel.

for both irregular types. The dust-to-metal ratio $D_{Z}^{\prime}$ observed in dwarf irregulars can be defined as:

$D_{Z}^{\prime}=\frac{M_{\text {dust }}}{M_{\mathrm{HI}} \cdot Z}=\frac{D^{\prime}}{Z}$.

In Sect. 3.1.2, we have seen that the dust depletion patterns (hence also the dust-to-metal ratio) are determined by the balance between destruction and accretion and that, in the S.N., it is impossible to derive constraints on the dust condensation efficiencies from the analysis of the observed depletion pattern. In dwarf irregulars, dust accretion is likely to play a negligible role or to be absent, meaning the variation in the condensation efficiencies has strong effects on the dust-to-metal ratio. In the right upper and lower panels of Fig. 16, we show the predicted evolution of $D_{Z}^{\prime}$ in dwarf irregular and starburst galaxies as a function of time and metallicity, respectively, calculated assuming the set of condensation efficiencies as suggested by D98 and a constant value of $\delta_{i}^{\mathrm{SW}}=\delta_{i}^{\mathrm{Ia}}=\delta_{i}^{\mathrm{II}}=0.1$. At variance with the S.N., adopting different condensation efficiencies has noticeable effects on the dust-to-metal ratio. In particular, the $D_{Z}^{\prime}$ values calculated by assuming the D98 condensation efficiencies are higher than the ones predicted by assuming a constant value of 0.1 .

In the lower right panel of Fig. 16, we show the predicted evolution of $D_{Z}^{\prime}$ as a function of the metallicity for the IC model and for the starburst model. By comparing the different predictions for $D_{Z}^{\prime}$, calculated assuming two different sets of dust condensation efficiencies, we can note that, in principle, the measure of the dust-to-metal ratio and of the dust depletion pattern in dwarf irregulars could allow one to put solid constraints on the dust condensation efficiencies.

\section{Conclusions}

By means of chemical evolution models for galaxies of different morphological types, we studied the cosmic evolution of the dust properties in different environments. By adopting the same formalism as developed by D98, we studied the evolution of the dust content of the S.N., confirming the main results of D98. We carried on a deep study of the space of the parameters used to model dust evolution and, thanks to the uptodate observations available in the solar vicinity, performed a fine-tuning of the parameters. In the following, we outline only the new aspects that were investigated here and that are different from the ones considered by D98. We extended our study to ellipticals and dwarf irregular galaxies, for which dust evolution was calculated by means of chemical evolution models relaxing the instantaneous recycling approximation, namely taking the stellar lifetimes into account. The main results concerning the present-day dust properties for the galaxies studied here are presented in Table 3. The various models are listed in Col. 1. In Cols. 2-6, we present the predicted present-day dust production rates, dust destruction rates, dust accretion rates, dust masses, dust-to-gas ratios and dust-to-metal ratios, respectively.

Our main results can be summarised as follows.

1. For each chemical element, we compared the predicted present-day fractions to the ones observed in the Local Interstellar Cloud. This analysis has been useful for testing the various parameters involved in our study, i.e. the dust condensation efficiencies $\delta_{i}^{\mathrm{SW}}, \delta_{i}^{\mathrm{Ia}}, \delta_{i}^{\mathrm{II}}$ and the dust destruction efficiency $\epsilon$. In the most realistic physical situation, i.e. once both the dust destruction and accretion processes are taken into account, the dust fractions are nearly independent of the choice of the dust condensation efficiencies for all the elements. The main processes determining the gas fractions are dust accretion and dust destruction. We can reproduce the observed dust fractions by assuming that the dust destruction efficiency depends on the properties of a given chemical element. A physical justification for this assumption might be found in the different condensation temperatures $T_{\mathrm{c}}$ of the elements. These assumptions allow us to also reproduce the gas and cosmic abundances observed in the Local Interstellar Cloud.

2. For all the elements, the evolution of the dust fractions is strongly determined by the SF history. At the beginning of each SF event, the dust fractions present a sharp peak, 
Table 3. Predicted present-day dust properties for the galactic models used in this work.

\begin{tabular}{|c|c|c|c|c|c|c|}
\hline Model & $\begin{array}{c}\text { DPR } \\
\left(M_{\odot} \mathrm{pc}^{-2} \mathrm{yr}^{-1}\right)\end{array}$ & $\begin{array}{c}\text { DDR } \\
\left(M_{\odot} \mathrm{pc}^{-2} \mathrm{yr}^{-1}\right)\end{array}$ & $\begin{array}{c}\text { DAR } \\
\left(M_{\odot} \mathrm{pc}^{-2} \mathrm{yr}^{-1}\right)\end{array}$ & $\begin{array}{c}\sigma_{\text {dust }} \\
\left(M_{\odot} \mathrm{pc}^{-2}\right)\end{array}$ & $\bar{D}$ & $\overline{D_{Z}}$ \\
\hline Milky Way, S.N. & 0.01 & 0.3 & 0.3 & 0.055 & 0.008 & 0.6 \\
\hline Milky Way, 16 kpc & $3.8 \times 10^{-5}$ & $1.3 \times 10^{-4}$ & $8.7 \times 10^{-5}$ & 0.008 & 0.0011 & 0.85 \\
\hline Model & $\begin{array}{c}\text { DPR } \\
\left(M_{\odot} \mathrm{yr}^{-1}\right)\end{array}$ & $\begin{array}{c}\text { DDR } \\
\left(M_{\odot} \mathrm{yr}^{-1}\right)\end{array}$ & $\begin{array}{c}\text { DAR } \\
\left(M_{\odot} \mathrm{yr}^{-1}\right)\end{array}$ & $\begin{array}{l}M_{\text {dust }} \\
\left(M_{\odot}\right)\end{array}$ & $\bar{D}$ & $\overline{D_{Z}}$ \\
\hline Elliptical, La1 & $2.5 \times 10^{-3}$ & $2.0 \times 10^{-3}$ & 0 & $0.7 \times 10^{6}$ & 0.0003 & 0.01 \\
\hline Elliptical, Ha1 & 0.024 & $2.0 \times 10^{-2}$ & 0 & $1.1 \times 10^{6}$ & 0.0003 & 0.011 \\
\hline Model & $\begin{array}{c}\mathrm{DPR} \\
\left(M_{\odot} \mathrm{yr}^{-1}\right)\end{array}$ & $\begin{array}{c}\text { DDR } \\
\left(M_{\odot} \mathrm{yr}^{-1}\right) \\
\end{array}$ & $\begin{array}{c}\text { DAR } \\
\left(M_{\odot} \mathrm{yr}^{-1}\right) \\
\end{array}$ & $\begin{array}{l}M_{\text {dust }} \\
\left(M_{\odot}\right) \\
\end{array}$ & $D^{\prime}$ & $D_{Z}^{\prime}$ \\
\hline Irregular, IC & $2 \times 10^{-4}$ & $2 \times 10^{-4}$ & 0 & $2 \times 10^{5}$ & $8 \times 10^{-4}$ & 0.16 \\
\hline Irregular, burst & $1.7 \times 10^{-3}$ & $1.0 \times 10^{-3}$ & 0 & $1.2 \times 10^{5}$ & $2 \times 10^{-4}$ & 0.24 \\
\hline
\end{tabular}

immediately followed by a very steep decrease, due to dust destruction by intense type II SN explosions.

During most of the cosmic time (i.e. from $\sim 3$ up to $10 \mathrm{Gyr}$ ) in the S.N. the dust fractions rise monotonically. The effect of the SF threshold causes rapid oscillations in the dust fractions only in the last 3 Gyr of evolution.

3. For the dust destruction rate, we predict present-day values of $\sim 5 \times 10^{-9} \mathrm{yr}^{-1}$ and $\sim 8 \times 10^{-9} \mathrm{yr}^{-1}$ for silicate and carbon dust, respectively. Similar values are predicted for the accretion rates for both dust types. Our values are in good agreement with the estimates by Draine (1990) and Tielens (1998) for a cold medium with different approaches.

4. For both type Ia and type II SNe, we compared the production and destruction rates and how these quantities vary throughout the evolution of the S.N. For type II SNe, dust destruction dominates dust production throughout almost all the cosmic history. On the other hand, for type Ia SNe during the first Gyr of galactic evolution, Si dust production has dominated dust destruction, but with negligible consequences on the total Si dust mass.

5. We studied the evolution of the dust-to-gas and dust-to-metal ratio as a function of time and metallicity in the S.N. and in the outermost regions of the Galactic disc. The disc outskirts evolve with an SF activity that is less intense than the S.N., with a lower present-day dust-to-gas ratio and a higher current dust-to-metal ratio. For both quantities, we compared the value predicted by means of the S.N. model to the one observed in the Galaxy, finding very good agreement.

6. In elliptical galaxies, type Ia $\mathrm{SNe}$ are the major dust factories in the past 10 Gyr. With our models, we successfully reproduce the dust masses observed in local ellipticals $\left(\sim 10^{6} M_{\odot}\right)$ by means of recent FIR and SCUBA observations.

7. We have shown that, by considering reduced dust destruction in a hot and rarefied medium, dust is helpful in alleviating the iron discrepancy observed in the hot gaseous halos surrounding local ellipticals (Arimoto et al. 1997). In this medium, we predict a $\mathrm{Fe}$ abundance of $[\mathrm{Fe} / \mathrm{H}]=0.15-0.6$ (depending on the adopted prescription for dust destruction) whereas Pipino et al. (2005) found $[\mathrm{Fe} / \mathrm{H}] \geq 0.85$ without dust. Moreover, the inclusion of dust improves the agreement between the predicted X-ray temperature $(0.7 \mathrm{keV})$ and luminosity $\left(\sim 10^{42} \mathrm{erg} \mathrm{s}^{-1}\right)$ for a model of a giant elliptical and the observations.

8. The dust masses observed in high-redshift SCUBA galaxies, the most likely progenitors of the local giant ellipticals, are successfully reproduced by our models, which predict up to $\sim 10^{8-9} M_{\odot}$ of dust during the high-redshift, star-forming phase. Furthermore, the dust treatment is very helpful in reproducing the abundances and the dust-to-gas ratios observed in Lyman Break Galaxies. In the specific case of the galaxy MS1512-cB58, we confirm the assumptions on dust made by Matteucci \& Pipino (2002) and reinforce their conclusions, i.e., that LBGs are very likely to be the progenitors of the local low-mass spheroids.

9. As for the metals, we predict that ellipticals play a major role in the dust enrichment of the IGM/ICM during galactic winds. A minor fraction (i.e. $\sim 10^{-4}$ of the dust mass ejected by ellipticals) is ejected by irregulars.

10. The two models used to study dust evolution in dwarf irregular galaxies present very different dust production rates. For the IC model (irregular galaxy with continuous star formation), the production rates behave smoothly throughout the full evolution of the system. For the starburst model, the evolution of the production rates reflects its intermittent SF history. Owing to the different SF histories, the starburst model presents higher dust fractions than the IC model at any time.

11. The predicted current dust-to-gas ratios for irregular galaxies are in general higher than the majority of the observed values. However, the determination of the dust mass in these galaxies is affected by several sources of uncertainty. By assuming a factor of 4 uncertainty in the observed data, as suggested by Lisenfeld \& Ferrara (1998), the observed dustto-gas ratios are consistent with our predictions.

12. In dwarf irregulars, dust accretion is likely to play a negligible role or to be absent, hence the variation of the condensation efficiencies has strong effects on the dust-to-metal ratio. In principle, a precise determination of the dust-tometal ratios and of the depletion pattern in dwarf irregular galaxies could be helpful for putting solid constraints on the dust condensation efficiencies.

13. Finally, it is interesting to compare our results for the current dust masses predicted for ellipticals and irregulars with the recent observational determinations, now possible thanks to the Spitzer Space Telescope. In local ellipticals, Kaneda et al. (2007) and Panuzzo et al. (2007) derive dust masses typically of the order of $10^{5}-10^{6} M_{\odot}$. The values computed by means of our fiducial model are compatible with the observationally derived values quoted above (see Table 3 ).

Also our predictions for the dust masses of dwarfs/irregular galaxies agree with the Spitzer results. In fact, for local dwarf galaxies, dust masses of $10^{4}-10^{6} M_{\odot}$ have been detected (Cannon et al. 2006; Walter et al. 2007), in perfect agreement 
with the values we predict for present-day dwarf galaxies, i.e. $1-2 \times 10^{5} M_{\odot}$.

Acknowledgements. We wish to thank Giovanni Vladilo, John Danziger, and Roberto Maiolino for many helpful discussions and suggestions.

\section{References}

Arimoto, N., \& Yoshii, Y. 1987, A\&A, 173, 23

Arimoto, N., Matsushita, K., Ishimaru, Y., Ohashi, T., \& Renzini, A. 1997, ApJ, 477, 128

Aguirre, A. 1999, ApJ, 525, 583

Awaki, H., Mushotzky, R., Tsuru, T., et al. 1994, PASJ, 46, L65

Bertin, G., Saglia, R. P., \& Stiavelli, M. 1992, ApJ, 384, 423

Bertoldi, F., Carilli, C. L., Cox, P., et al. 2003, A\&A, 406, L55

Bradamante, F., Matteucci, F., \& D’Ercole, A. 1998, A\&A, 337, 338

Buote, D. A. 1999, MNRAS, 309, 685

Buote, D. A., \& Fabian, A. C. 1998, MNRAS, 296, 977

Calura, F., \& Matteucci, F. 2006a, MNRAS, 369, 465

Calura, F. \& Matteucci, F. 2006b, ApJ, 652, 889

Calura, F., Matteucci, F., \& Vladilo, G. 2003, MNRAS, 340, 59

Cannon, J. M. 2006, ApJ, 652, 1170

Chiappini, C., Matteucci, F., \& Gratton, R. 1997, ApJ, 477, 765

Chiappini, C., Matteucci, F., \& Romano, D. 2001, ApJ, 554, 1044

Clayton, G. C., Green, J., Wolff, M. J., et al. 1996, ApJ, 460, 313

Dessauges-Zavadsky, M., Calura, F., Prochaska, J. X., D’Odorico, S., \& Matteucci, F. 2004, A\&A, 416, 79

Dickey, J. M. 1993, in The Minnesota Lectures on the Structure and Dynamics of the Milky Way, ed. R. M. Humphreys (San Francisco: ASP), ASP Conf Ser., 39, 93

Draine, B. T. 1981, ApJ, 245, 880

Draine, B. T. 2003, ARA\&A, 41, 241

Draine, B. T. 1990, in The Evolution of the Interstellar Medium, ed. L. Blitz (San Francisco: ASP), ASP Conf. Proc., 12, 193

Draine, B. T., \& Salpeter, E. E. 1979, ApJ, 231, 438

Dunne, L., Eales, S. A., \& Edmunds, M. G. 2003, MNRAS, 341, 589

Dwek, E. 1998, ApJ, 501, 643 (D98)

Eales, S., Lilly, S., Webb, T., et al. 2000, AJ, 120, 2244

Edmunds, M. G. 2001, MNRAS, 328, 223

Ferrarotti, A. S., \& Gail, H.-P. 2006, A\&A, 447, 553

Flagey, N., Boulanger, F., Verstraete, L., et al. 2006, A\&A, 453, 969

François, P., Matteucci, F., Cayrel, R., et al. 2004, A\&A, 421, 613

Giavalisco, M. 2002, ARA\&A, 40, 579

Greenberg, J. M., \& Li, A. 1999, Adv. Space Res., 24, 497

Greggio, L., \& Renzini, A. 1983, A\&A, 118, 217

Hirashita, H. 1999, ApJ, 522, 220

Hirashita, H., Tajiri, Y. Y., \& Kamaya, H. 2002, A\&A, 388439

Holweger, H. 2001, AIPC, 598, 23

Hughes, D., et al. 1998, Nature, 394, 241

Humphrey, P. J., \& Buote, D. A. 2006, ApJ, 639, 136

Inoue, A. K. 2003, PASJ, 55, 901

Issa, M. R., MacLaren, I., \& Wolfendale, A. W. 1990, A\&A, 236, 237

Itoh, H. 1989, PASJ, 41, 853

Iwamoto, K., Brachwitz, F., Nomoto, K., et al. 1999, ApJS, 125, 439I

Jones, A. P., \& Tielens, A. G. G. M. 1994, The Cold Universe, ed. Th. Montmerle, Ch. J. Lada, I. F. Mirabel, \& J. Tran Thanh Van (Gif-sur-Yvette: Éditions Frontières), 35

Jones, A. P., Tielens, A. G. G. M., Hollenbach, D. J., \& McKee, C. F. 1994, ApJ, 433, 797
Jones, A. P., Tielens, A. G. G. M., \& Hollenbach, D. J. 1996, ApJ, 469, 740 Kaneda, H., Onaka, T., Kitayama, T., Okada, Y., \& Sakon, I. 2007, PASJ, 59, 107

Kennicutt, R. C. 1989, ApJ, 344, 685

Kennicutt, R. C. 1998, ApJ, 498, 541

Kimura, H., Mann, I., \& Jessberger, E. K. 2003, ApJ, 582, 846

Kulkarni, S. R., \& Heiles, C. 1987, in Interstellar Processes, ed. D. Hollenbach, \& H. Thronson (Dordrecht: Kluwer), 87

Lanfranchi, G., \& Matteucci, F. 2003, MNRAS, 345, 71, LM03

Leeuw, L. L., Sansom, A. E., Robson, E. I., Haas, M., \& Kuno, N. 2004, ApJ, 612,837

Li, A. 2005, ApJ, 622, 965

Li, A., \& Greenberg, J. M. 1997, A\&A, 323, 566

Lilly, S. J., Eales, S. A., Gear, W. K. P., et al. 1999, ApJ, 518, 641

Lisenfeld, U., \& Ferrara, A. 1998, ApJ, 496, 145

Lodders, K. 2003, ApJ, 591, 1220

Maiolino, R., Schneider, R., Oliva, E., et al. 2004, Nature, 431, 533

Mathis, J. S. 1996, ApJ, 472, 643

Matsumoto, H., Koyama, K., Awaki, H., et al. 1997, ApJ, 482, 133

Matteucci, F. 1992, ApJ, 397, 32

Matteucci, F. 1994, A\&A, 288, 57

Matteucci, F., \& François, P. 1989, MNRAS, 239, 885

Matteucci, F., \& Greggio, L. 1986, A\&A, 154, 279

Matteucci, F., \& Pipino, A. 2002, ApJ, 569, L69

Matteucci, F., \& Recchi, S. 2001, ApJ, 558, 351

Matteucci, F., \& Tornambé, A. 1987, A\&A, 185, 51

Matteucci, F., Renda, A., Pipino, A., \& Della Valle, M. 2003, A\&A, 405, 23

McKee, C. F. 1989, in Interstellar Dust, ed. L. J. Allamandola, \& A. G. G. M. Tielens (Dordrecht: Kluwer), Proc. IAU Symp., 135, 431

Morgan, H. L., \& Edmunds, M. G. 2003, MNRAS, 343, 427

Nomoto, K., Iwamoto, K., Nakasato, N. T., et al. 1997b, Nucl. Phys. A, 621, 467

Panuzzo, P., Vega, O., Bressan, A., et al. 2007, ApJ, 656, 206

Pettini, M., Shapley, A. E., Steidel, C. C., et al. 2001, ApJ, 554, 981

Pettini, M., Rix, S. A., Steidel, C. C., et al. 2002, ApJ, 569, 742

Pipino, A., \& Matteucci, F. 2004, MNRAS, 347, 968

Pipino, A., Matteucci, F., Borgani, S., \& Biviano, A. 2002, New Astron., 7, 227

Pipino, A., Kawata, D., Gibson, B. K., \& Matteucci, F. 2005, A\&A, 434, 553

Popescu, C. C., Tuffs, R. J., Voelk, H. J., Pierini, D., \& Madore, B. F. 2002, ApJ, 567,221

Portinari, L., Chiosi, C., \& Bressan, A. 1998, A\&A, 334, 505

Rana, N. C. 1991, ARA\&A, 29, 129

Recchi, S., Matteucci, F., D’Ercole, A., \& Tosi, M. 2002, A\&A, 384, 799

Renzini, A., \& Voli, M. 1981, A\&A, 94, 175

Romano, D., Chiappini, C., Matteucci, F., \& Tosi, M. 2005, A\&A, 430, 491

Salpeter, E. E. 1955, ApJ, 121, 161

Savage, B. D., \& Sembach, K. R. 1996, ARA\&A, 34, 279

Scalo, J. M. 1986, FCPh, 11, 1

Schmidt, M. 1959, ApJ, 129, 243

Shull, J. M. 1978, ApJ, 226, 858

Talbot, R. J., \& Arnett, W. D. 1971, ApJ, 170, 409

Temi, P., Brighenti, F., Mathews, W. G., Bregman, J. D.

Teplitz, H. I., et al. 2000, ApJ, 533, L65

Thornton, K., Gaudlitz, M., Janka, H.-T., \& Steinmetz, M. 1998, ApJ, 500, 95

Tielens, A. G. G. M. 1998, ApJ, 499, 267

Tilton, G. R. 1988, in Meteorites and the early solar system (University of Arizona Press), 259

van den Hoek, L. B., \& Groenwegen, M. A. T. 1997, A\&AS, 123, 305

Vlahakis, C., Dunne, L., Eales, S. 2005, MNRAS, 364, 1253

Walter, F., et al. 2007, ApJ, 661, 102

Woosley, S. E., \& Weaver, T. A. 1995, ApJS, 101, 181

Zhukovska, S., Gail, H.-P., \& Trieloff, M. 2007, A\&A [arXiv:0706. 1155] 